\title{
New light on the mycological work of Lewis David von Schweinitz:
}

\section{A volume of illustrations for the Conspectus fungorum at the Farlow Reference Library of Harvard University}

\author{
Jason M. Karakehian ${ }^{1}$, William R. Burk², and Donald H. Pfister ${ }^{1}$ \\ ${ }^{1}$ Farlow Herbarium of Harvard University, 22 Divinity Avenue, Cambridge, MA 02138, USA: corresponding author e-mail: jkarakehian@gmail.com \\ ${ }^{2}$ Department of Biology, University of North Carolina, CB no. 3280 Coker Hall, Chapel Hill, NC 27599-3280, USA
}

Abstract: In 1993, the Farlow Reference Library of Cryptogamic Botany at Harvard University received a gift of a slim volume, entitled Icones fungorum Niskiensium. Vibrant water colour illustrations of fungi covered the pages bound within it. It was attributed to Lewis David von Schweinitz, the American born "father" of North American mycology. Schweinitz coauthored his first mycological publication with Johannes Baptista von Albertini in Germany, 1805: Conspectus fungorum in Lusatiae superioris agro Nieskiensi crescentium (An overview of fungi growing in the area of Niesky in Upper Lusatia), now considered a classic mycological text. Schweinitz, a prolific and skillful mycological illustrator, prepared the plates of illustrations appended to the Conspectus and produced many unpublished water colours of the fungi treated therein. The results of our research into the provenance of the Icones fungorum Niskiensium are presented here. Building upon recent scholarship, we provide analysis of its role in the early development of the Conspectus. An index to the figures, cross-referenced to Schweinitz's other unpublished volumes of water colours and to the Conspectus, is also provided. To make the Icones fungorum Niskiensium publically available, the volume has been digitized and may be accessed through the Biodiversity Hertitage Library (BHL) portal.

Key words: mycological illustration, mycological history, fungi, Moravian Church, nineteenth century science, mycobiota, female botanists, bibliographic sleuthing

\section{INTRODUCTION}

In the archives of the Farlow Reference Library of Cryptogamic Botany at Harvard University there is a slim volume bound in red cloth, consisting of 249 vibrant water colour paintings of various species of fungi. The name of the artist is not to be found on any page, though the title: Icones fungorum Niskiensium (hereafter referred to as the Harvard Icones), along with the distinctive script of the scientific names that are written-in beside each illustration, held clues that led one of us (D.H.P.) to connect the volume to a well-known figure in the history of mycology - the "father" of North American mycology, Lewis David von Schweinitz (1780-1834) (Rogers 1977).

Born in Bethlehem, Pennsylvania, Schweinitz's profession was as a minister and administrator in the Moravian Church in America, a mainline Protestant denomination that became established in Pennsylvania in 1741 (Hamilton 1900, Rogers 1977). His life-long avocation in botany is said by one contemporary biographer to have begun when he was a "mere child" with a chance encounter with a specimen of Lichen digitatus (now Cladonia digitata) on display in Nazareth Hall, a local Moravian school where Schweinitz was visiting and was later enrolled as a pupil at the age of seven. Amid his other ecclesiastical studies, he enthusiastically pursued botanical training under the tutelage of one of his teachers, the botanist Samuel Gottlieb Kramsch (1756-1824) (Johnson 1835, Wilbert 1908, Rogers 1977). At the age of eighteen, in 1798 , he attended a seminary in Niesky, Germany (roughly east of Leipzig, near the Polish border) where he soon began to devote his spare time to collecting, painting and identifying fungi with his associates. There, in 1805 , he published in collaboration with his friend, professor and mentor Johannes Baptista von Albertini (1769-1831), an account of the fungi of that locality entitled Conspectus fungorum in Lusatiae superioris agro Nieskiensi crescentium. E methodo Persooniana [An overview of fungi growing in the area of Niesky in Upper Lusatia, according to the methodology of Persoon] (hereafter referred to as the Conspectus) (Rogers 1977, Hewitt et al. 2016). It documents more than 1000 species, including over 100 new species attributed to "Alb. \& Schwein." These are organized according to the systematic framework of Christiaan Hendrik Persoon (1761-1836) as outlined in his 1801 Synopsis methodica fungorum. The Conspectus was well- received by European mycologists, though it was noted by an early reviewer that, to make full use of it, a worker would also require Persoon's Synopsis (Anon. 1806). Furthermore, the Conspectus was important to the scientific development of Elias Magnus Fries (1794-1878) who erected an overarching framework classification for fungi and whose Systema mycologicum (1821-32) is now a sanctioning work (Rogers 1977, Hewitt et al. 2016).

The Conspectus is now considered a classic mycological text and also recognized as an ecological record (Hewitt et al. 2016). It includes 12 hand-coloured plates that Schweinitz had drawn. Each consists of between six and ten figures that depict a selection of the new species described in the text. To create accurate and informative illustrations, Schweinitz drew from recently collected, fresh specimens. However, when these were not available, he referred to fungarium specimens and to a number of volumes wherein he had taken great care to paint detailed water colour portraits of representative specimens. Fleshy fungi, such as agarics and boletes, presumably would have been painted while the specimens were fresh as their shape and colouration change dramatically when dried. Thus, these illustrations served as field notes for how 
Table 1. A list of Lewis David von Schweinitz's volumes of unpublished water colour paintings of fungi, with Albertini and Schweinitz's Conspectus. The five-volume set and the Harvard Icones were created by Schweinitz and were used as reference materials in the development of the Conspectus. Volume 4 is a supplement to volume 1 and volume 5 is a supplement to volumes 2 and 3 (Hewitt 2002). Note the variation in the spelling of the title between volumes 1-3 and 4, 5 . Volume 1 contains plates numbered 1-77, but lacks plates 33, 40, 44-47, 50, 51. Nearly every species listed in the Conspectus is illustrated in the five-volume set. The Harvard Icones is a sketchbook of illustrations that are preliminary to the finished paintings in the five-volume set and the etched plates in the Conspectus. The Michigan Icones are water colour copies of figures from volumes 2, 3 and 5 of the five-volume set and were executed in America. Vol.: Volume number of the five-volume set. Dates: our approximation of when each volume was created. Our rationale is outlined in Supplementary Table S1. Abbrev. name: an abbreviation of the volume name used here. No. pl. and No. figs.: the number of plates and figures in the volume, respectively, with totals for the five-volume set given in italics. MS loc.: manuscript location, given as an abbreviation of the names of the following institutions: ANS: Archives of the Academy of Natural Sciences of Drexel University, Philadelphia, Pennsylvania. UNC: Rare Book Collection, Wilson Library, University of North Carolina, Chapel Hill. H: Farlow Reference Library of Cryptogamic Botany, Harvard University, Cambridge, Massachusetts. M: Herbarium Library, University of Michigan, Ann Arbor, Michigan. NOTE: all volumes except for the Michigan Icones are available online in digitized form, see References.

\begin{tabular}{|c|c|c|c|c|c|c|}
\hline Vol. & Title & Dates & Abbrev. name & No. pl. & No. figs. & MS loc. \\
\hline 1 & Fungorum Nieskiensium Icones & (ca.) 1802-1803 & \multirow{5}{*}{ Five-volume set } & 69 & 199 & ANS \\
\hline 2 & Fungorum Nieskiensium Icones & (ca.) 1802-1803 & & 97 & 242 & ANS \\
\hline 3 & Fungorum Nieskiensium Icones & 1803 & & 99 & 267 & ANS \\
\hline 4 & Fungorum Niskiensium Icones & (ca.) 1803-1805 & & 76 & 201 & UNC \\
\hline 5 & Fungorum Niskiensium Icones & (ca.) 1803-1805 & & 104 & 242 & ANS \\
\hline NA & Icones Fungorum Niskiensium & (ca.) 1798-1802 & Harvard Icones & 50 & 249 & $\mathrm{H}$ \\
\hline NA & Icones Fungorum & (ca.) 1818-1826 & Michigan Icones & 301 & 716 & M \\
\hline NA & $\begin{array}{l}\text { Conspectus fungorum in Lusatiae } \\
\text { superioris agro Nieskiensi crescentium. } \\
\text { E methodo Persooniana. }\end{array}$ & 1805 & Conspectus & 12 & 94 & NA \\
\hline
\end{tabular}

the fungi appeared in nature (Hewitt et al. 2016). Seven volumes of original water colours are known to exist, and these are held in various American institutions. Table 1 lists their titles and locations, among other information, and provides a note on the relationship of each to the Conspectus. Scholarship on these volumes and the Conspectus includes: Shear \& Stevens (1917), Krieger (1938-39), Lynch (1996), Hewitt (2002), and Hewitt et al. (2016).

Attribution of the Harvard Icones by Pfister allowed Hewitt (2002) to critically examine its relationship to the four volumes held in the Archives of the Academy of Natural Sciences of Drexel University, Philadelphia (ANS), though a thorough investigation of its provenance was beyond the scope of his research. He determined that five of the volumes formed a distinct set, and that the Harvard Icones and the Michigan Icones were separate from this (Table 1). He hypothesized that the Harvard Icones served Schweinitz as an early sketchbook (D. Hewitt, pers. comm. August 2000).

As part of our efforts to learn more about how these unpublished volumes might have served Schweinitz in his mycological work and what their relationships to the Conspectus might be, we indexed the more than 1000 species listed in the Conspectus and crossreferenced them with our index of all of the species figured in the seven volumes of unpublished illustrations. Additionally, we visually compared all of the figures from the Conspectus plates with the respective figures of each of these species that are in the unpublished volumes. We discovered that nearly every species listed in the Conspectus has a corresponding illustration in a volume of the five-volume set. The quality of these figures, being larger format and original paintings, are generally superior to the engravings in the Conspectus. We created a master index of figures for each species listed in the Conspectus. We also identified figures from the Conspectus plates that Schweinitz might have directly copied or otherwise adapted from his unpublished illustrations. The full results of this research will be presented in a future publication. As the Harvard Icones is unique among the volumes of Schweinitz's paintings of fungi, and given the complicated history of its ownership, we will treat the Harvard Icones separately here.

Hewitt (2002) provides a description of the Harvard Icones with some notes on provenance. In order to facilitate our discussion, however, a re-description of the volume is provided here. The complete volume has been digitized and made available to the public online through the Biodiversity Heritage Library (BHL).

The format of the volume is $26 \mathrm{x}$ $19 \mathrm{~cm}$, with cover boards bound in red cloth. The title ICONES FUNGORUM NISKIENSIUM is centered on the front board in stamped gold leaf type (Fig. 1A). There is no title printed on the spine. On the reverse front cover, the pastedown endpaper bears pencil markings and three book plates (Fig. 1B). The pencil markings include "original water-colours of American fungi" and some illegible notations. One of the book plates is placed in the upper left corner and is $1.3 \times 2.2 \mathrm{~cm}$. It is printed on pink paper and reads: "W. Webster. Bookseller \& Stationer. Late G. Fell. 60, Piccadilly." (Fig. 1C). A second book plate measuring $10 \times 7.5 \mathrm{~cm}$ is centered on the pastedown and inscribed: "Rare Book S4134. Farlow Reference Library of Cryptogamic Botany. Harvard University. Gift of Elio Schaechter. Friends of the Farlow." (Fig. 1B). This book plate can be lifted from the bottom to reveal another book plate, $8 \times 6 \mathrm{~cm}$. It is inscribed with arms, a motto in Latin "ORA E E SEMPRE" and the name "Sir Francis Denys Bart." (Fig. 1D). After the free endpaper, there is a single fly-leaf bearing a hand-written inscription in ink on the recto: "Catherine Eliza Perceval - Philadelphia - March $8^{\text {th }} 1826$-.” (Fig. 1E). Following this are 50 leaves of plates, 25 $\mathrm{x} 18 \mathrm{~cm}$, each bearing several water colour figures of fungi on the recto with a plate number written in the upper right corner (Fig. 2). Several figures throughout the volume are annotated in pencil with nonconsecutive numbers that are enclosed or not in parentheses. There are 249 figures in total. Most are labeled with a Latin binomial scientific name in ink, by Schweinitz. Three are labeled Nobis [ours], denoting 


\section{ICONES}

\section{FUNGORU M NISKIENSIUM}

$$
\text { 5. }
$$

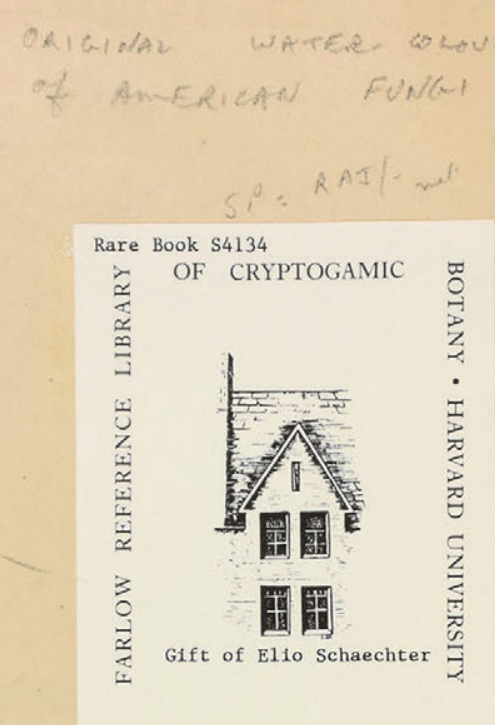

FRIENDS of the FARLOW

B
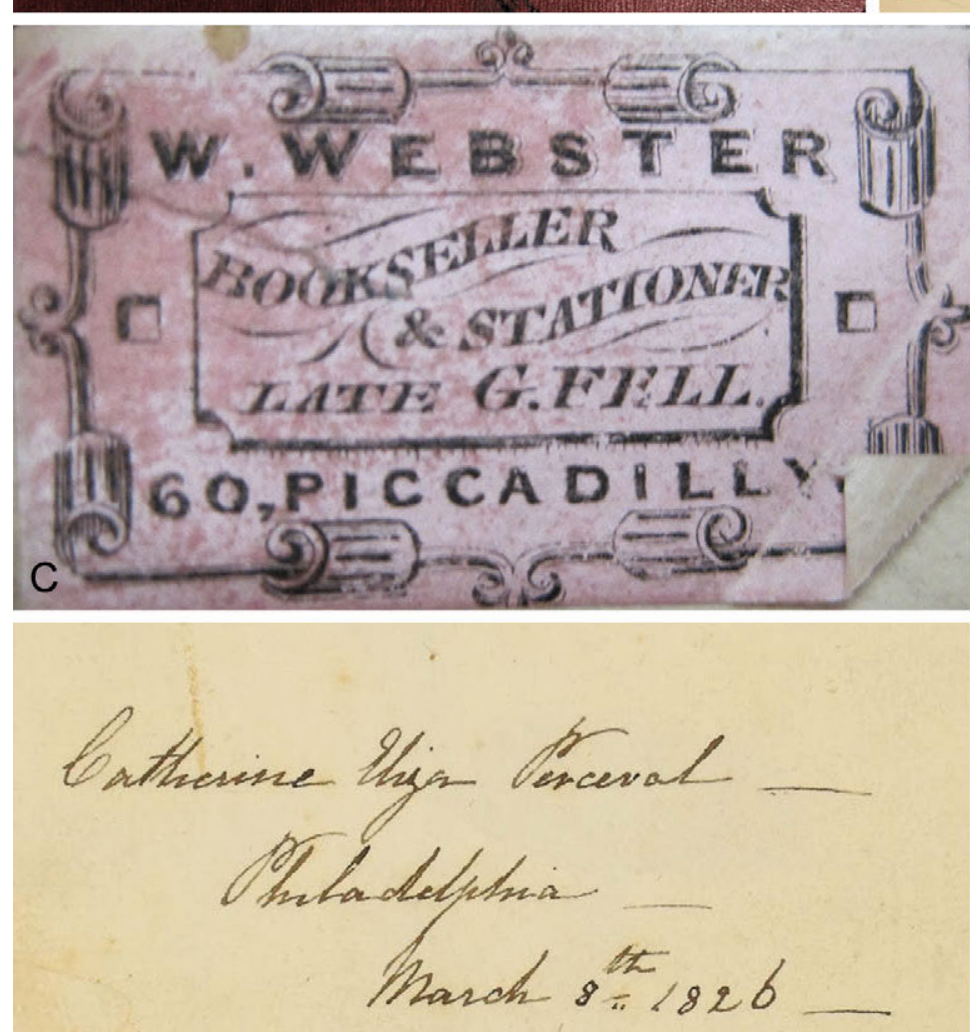

E

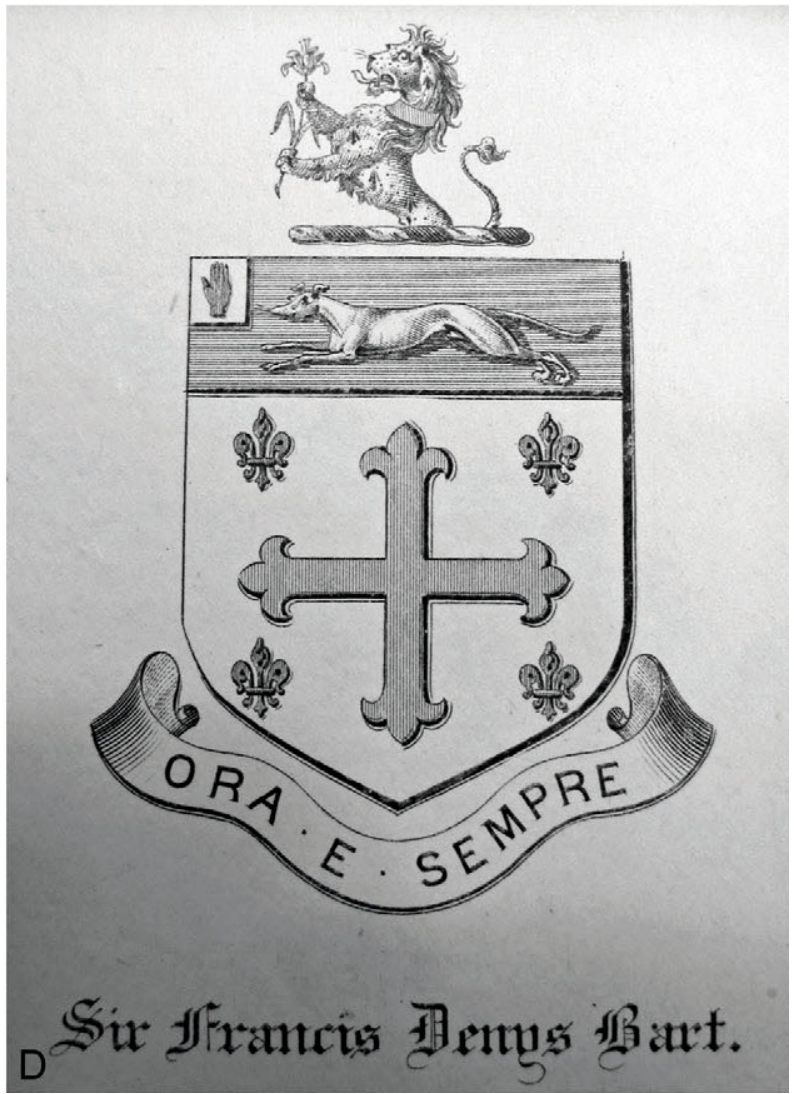

Fig. 1. Details of the Harvard Icones. A. Front cover. B. Reverse front cover, pastedown endpaper with bookplates and markings in pencil; the centre bookplate is attached at the top edge and can be lifted from the bottom to reveal another bookplate that is figured in D. C. Enlarged view of bookseller's plate in the upper left corner of B. D. Hidden bookplate that is beneath the centre bookplate in B. E. Inscription on the fly-leaf. Images courtesy of the Farlow Reference Library of Cryptogamic Botany, Harvard University. 


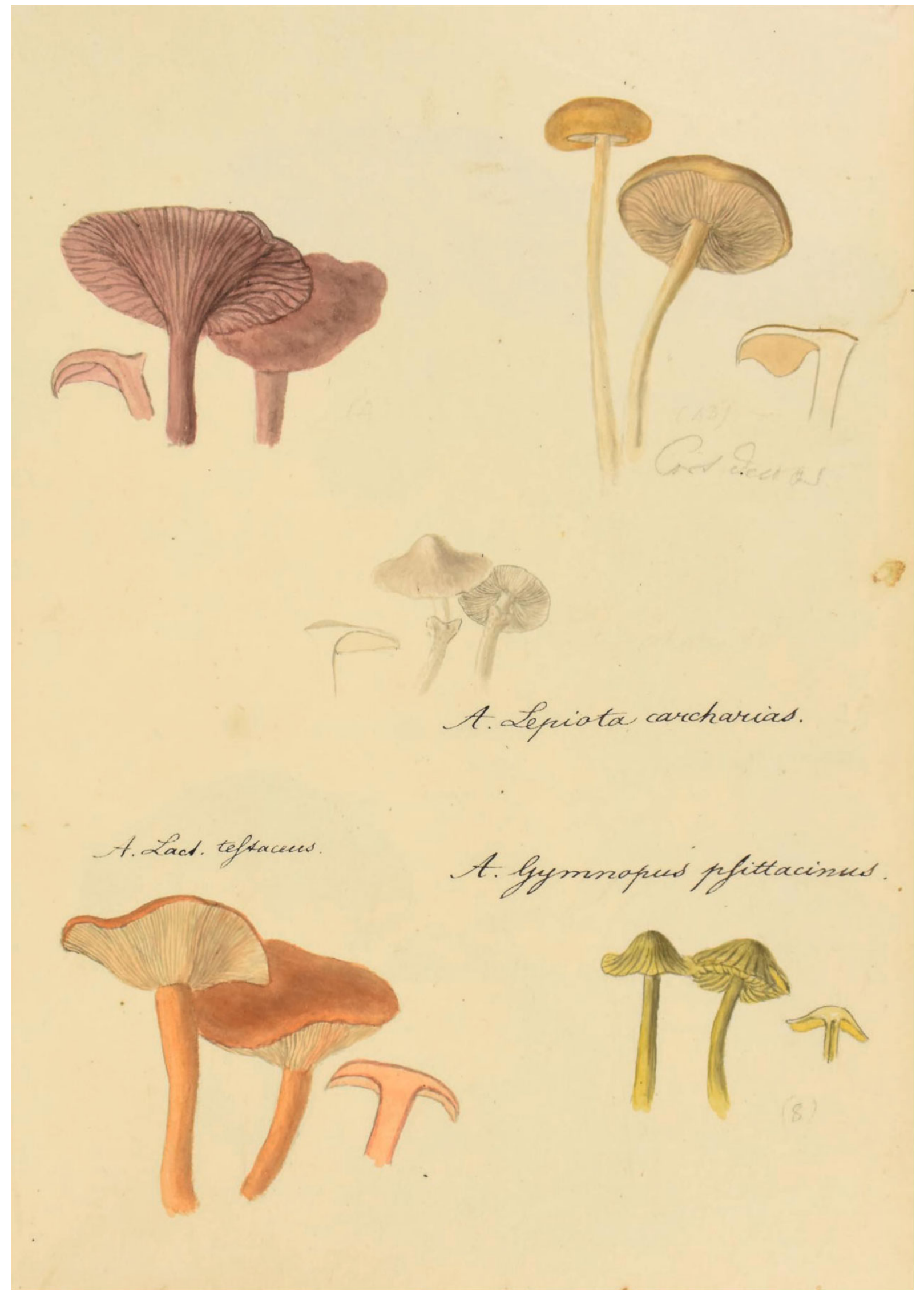

Fig. 2. A representative plate from the Harvard Icones depicting various species of gilled mushrooms in water colour (adapted from plate 47). Each figure shows two mushrooms in the round with a reduced insert showing one in longitudinal section through the center. Though most of the figures throughout the Harvard Icones are identified, a handful are not (upper left). Some figures have tentative identifications written in faint pencil (upper right). Others have numbers written in faint pencil beside them (upper and lower right). To what the numbers refer to remains unknown. Upper left: unidentified gilled mushroom. Upper right: "Cort. decoras", “(48)”. Centre: Agaricus (Lepiota) carcharias. Lower left: Agaricus (Lactifluus) testaceus. Lower right: Agaricus (Gymnopus) psittacinus, “(8)”. Image courtesy of the Farlow Reference Library of Cryptogamic Botany, Harvard University. 
new species that were described in the Conspectus: Peziza rhizophora, Poria cornuta (as Ceratium porioides), and Mesenteriaca grisea. Other than Perceval's inscription, the volume is unsigned. On some of the leaves page numbers, figures, and names near the edges have been cropped.

\section{RESULTS}

Table 2 is an index to all of the figures in the Harvard Icones. It is adapted from an unpublished index prepared by D.H.P. in August 1993 archived along with the volume. Except for the three names marked as new species, nearly all were established prior to the publication of the Conspectus and are treated in Persoon (1801). To facilitate use, we provide standardized names following Persoon (1801), with verbatim names given secondly. Page numbers are given where each taxon is treated in Persoon (1801) and in the Conspectus. Current names are not given. Illustrations without names are listed at the end of the index. Each name is crossreferenced to other illustrations of the same taxon in the Conspectus, the five-volume set, and to the Michigan Icones. Schweinitz's annotations and notes are copied verbatim. Our observations are given as comments.

\section{DISCUSSION}

The first part of this discussion relates to the provenance of the Harvard Icones and it proceeds in a roughly chronological sequence with section headings based on the bookplates and pencil markings on the pastedown endpaper, as well as Perceval's inscription on the fly-leaf. The second part is a discussion of how the illustrations served as sketches for Albertini and Schweinitz's reference in the development of the Conspectus, as well as how these relate to Schweinitz's other volumes of illustrations.

\section{Notes on the provenance of the Harvard icones}

\author{
Inscription: Catherine Eliza Perceval - \\ Philadelphia - March $8^{\text {th }} 1826-$ (Fig. \\ 1E).
}

Catherine Eliza Perceval (1811-1884) was born on 25 September in Québec (Morgan
1903) to the English-born Anne Mary Perceval (née Flower, 1790-1876) and Michael Henry Perceval (1779-1829). Soon after their marriage in 1810, the Percevals emigrated to Canada when M. Perceval was made Collector of Customs for Québec. In 1815 he established "Spencer Wood," an estate in Sillery, just west of Québec City (Pringle 1985), where C. Perceval grew into a young woman. She was the eldest daughter of ten children (Morgan 1903). The Percevals were members of a "Château Clique" of powerful and wealthy families that owned magnificent estates in the outskirts of Québec City (Creese 2010). Anne Perceval educated C. Perceval and her siblings in English, French, Italian, and Latin as well as in music and drawing. Self-reliance was valued. A family associate noted that "Instead of fancy work the young ladies were taught to repair their clothes and do plain sewing; this did not prevent them from making most brilliant matches" (LeMoine 1882). Through the period of 1820-28, A. Perceval's interest was drawn to botany through her association with two other distinguished local wives: Christian Ramsay (1786-1839), the Countess of Dalhousie, already an accomplished botanist, and Harriet Campbell Sheppard (fl. 1820s-30s); the husbands of whom also shared interests in natural history, amassing large personal libraries (Pringle 1985, Creese 2010). The trio collected extensively in Spencer Wood, the Ramsay and Sheppard estates, and in the estates of friends. They botanized in a variety of habitats including bogs and swamps (Pringle 1985). Considering the customs of the time, it is unclear how they conducted the often dirty and laborious practice of botany, though it was already established as an acceptable pastime for young and adult women in late $18^{\text {th }}$ century Britain and America (Keeney 1992). There are indications of A. Perceval's freedom regarding botanizing in her correspondence with William Darlington (1782-1863) and John Torrey (1796-1873) in which she discusses sharing botanical literature, collecting, and mounting specimens (Perceval 1826, Perceval, n.d.). The women built personal herbaria and shared specimens with correspondents such as William Jackson Hooker (1785-1865) as well as Darlington and Torrey. Pringle (1985) notes that "Mrs. Perceval" in particular is listed as a collector in Hooker's Flora boreali-americana ([1829]-1840) and in Torrey \& Gray's A flora of North America (1838-43). About 100 of her specimens were in Schweinitz's herbarium, though it is unclear if he received them directly from her.

In regards to cryptogamic botany, A. Perceval recounted in a letter to Torrey (Perceval n.d.) that her botanical interests ranged into mosses, ferns, lichens and fungi, and that she desired to send him quality specimens of these. She had received bryological literature from him, and was working with lichens and ferns "without either Guide or Specimen.” Fungi gave "considerable trouble from the circumstance of not knowing how to preserve them best..., but her scientific spirit was intrepid and ambitious: "to the whole of Cryptogamia Mrs. Perceval will now bestow considerable attention..."

Catherine Perceval came to possess the Harvard Icones as a result of her mother's botanical interests and network of correspondents. A letter from Darlington to A. Perceval dated 10 June 1826 indicates that they had met recently at the home of their mutual friend "Mr. Steinhauer" in Philadelphia (Darlington 1826). If C. Perceval's inscription in the Harvard Icones served for her as a reminder of the date and place that she received the volume, then a more precise date of the meeting would be 8 March. Joseph Daniel Steinhauer (17851852), known as Daniel, was a teacher and botanist. Darlington was introduced to Daniel by Schweinitz who regarded him as a "most valued \& excellent friend" and "an excellent botanist $\&$ a man of sound science generally" (Schweinitz 1825). How Daniel became acquainted with Schweinitz is unknown, but as both men were botanists, it is likely that Daniel's brother Henry played a central role in their meeting (Stuckey 1966). Rev. Henry Steinhauer (1782-1818) was a botanist, paleobotanist, and principal of the Moravian Seminary for Young Ladies at Bethlehem (PA) prior to his death. Both brothers were English-born (Stuckey 1966, Torrens 2005). Schweinitz's friendship with $\mathrm{H}$. Steinhauer dates to his early days at the Moravian seminary in Germany. He is described along with Albertini as "cherished associates” by Schweinitz's contemporary and biographer Walter R. Johnson (1835). Albertini and Schweinitz also acknowledge him in the introduction to the Conspectus as having assisted in the collection of fungi at Niesky. A letter from H. Steinhauer (Steinhauer 1810) outlining his role in the development of the Conspectus is transcribed in Hewitt et al. (2016). He departed the seminary in 1801 , his education completed 
Table 2. Index to the figures in the Harvard Icones. Current names are not given. Abbreviations. Cfr. $=$ confer. Sp. $=$ one species. Spp. $=$ two or more species. Schweinitz would sometimes abbreviate double m's or n's by writing one letter with a bar over it. An example here is Agaricus (Cortinaria) cinnamomeus.

Standardized name is according to Persoon (1801) with group given in parentheses. Pers. 1801 is the page number where the taxon is treated in Persoon (1801). Plate is the plate number where the taxon is figured in Harvard Icones. Each plate number is preceded by "H." This convention is continued in listings for "Other figures." Verb. name is the verbatim name inserted beside the figure by Schweinitz. Verb. no. is verbatim number - any legible numbers written in pencil beside the figure. The numbers are non-contiguous and non-repeating. What they refer to is currently unknown. Other figures includes references for other illustrations of this taxon in the five-volume set, the Harvard Icones, the Michigan Icones and the Conspectus. Refer to Table 1 for more information on these volumes. Figure references are given in the following formats: the five-volume set [volume number: plate number: figure number]; Harvard Icones $[\mathrm{H}+$ plate number]; Michigan Icones $[\mathrm{M}+$ plate number] (N.B. Figures in the Michigan Icones are copies of figures from volumes 2, 3 and 5 of the five-volume set); Conspectus [C + plate number: figure number]. In the five-volume set, each volume contains two indices: Index A fungorum and Index B tabularum. The former is indexed alphabetically by generic name and the latter is indexed numerically by table, then figure number. Conspectus is the page number where the taxon is treated in the Conspectus. Verb. notes are verbatim notes by Schweinitz. Comments are our notes and observations given as footnotes.

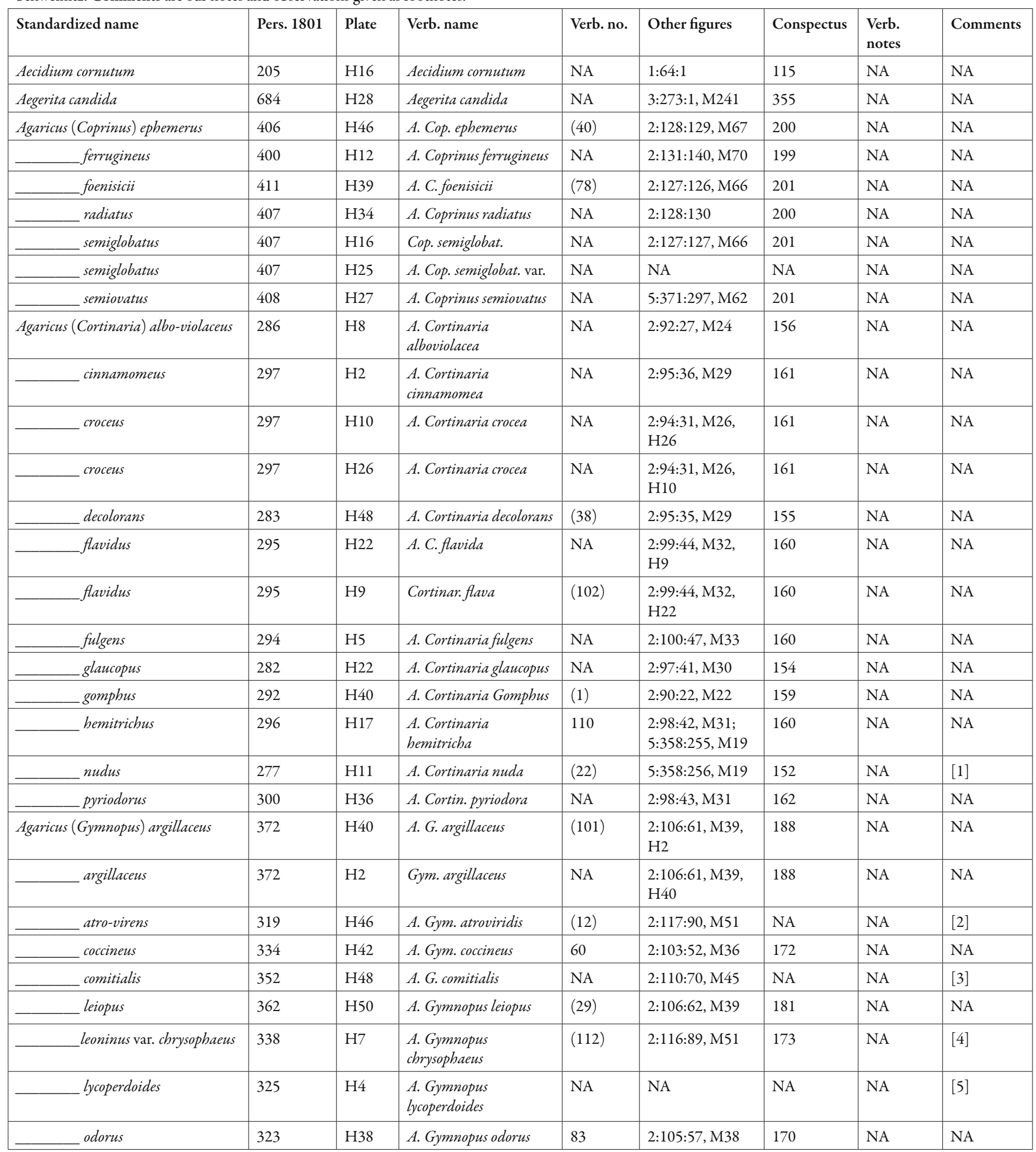


Table 2. (Continued).

\begin{tabular}{|c|c|c|c|c|c|c|c|c|}
\hline Standardized name & Pers. 1801 & Plate & Verb. name & Verb. no. & Other figures & Conspectus & $\begin{array}{l}\text { Verb. } \\
\text { notes }\end{array}$ & Comments \\
\hline _ platyphyllus & 362 & $\mathrm{H} 23$ & $\begin{array}{l}\text { A. Gymnopus } \\
\text { platyphylla a }\end{array}$ & NA & 2:113:77, M42. & 180 & NA & [6] \\
\hline _pluteus & 357 & $\mathrm{H} 13$ & A. Gymnopus pluteus. & NA & $2: 119: 95$ & 180 & NA & NA \\
\hline pratensis & 304 & $\mathrm{H} 31$ & A. Gymnopus pratensis & $(47)$ & 2:109:69, M45 & 162 & NA & NA \\
\hline _psittacinus & 335 & $\mathrm{H} 47$ & A. Gymnopus psittacinus & (8) & 2:104:56, M37 & 172 & NA & {$[7]$} \\
\hline purus & 339 & $\mathrm{H} 7$ & A. Gymnopuspurus & NA & $2: 107: 64, M 40$ & 173 & NA & NA \\
\hline ramealis & 375 & $\mathrm{H} 1$ & $\begin{array}{l}\text { A. Gymnopus ramealis. } \\
\text { Omph. epiphylla }\end{array}$ & NA & 2:114:81, M43 & 190 & NA & NA \\
\hline russula & 338 & $\mathrm{H} 10$ & A. Russula. & NA & NA & NA & NA & NA \\
\hline vaccinus & NA & $\mathrm{H} 15$ & A. Gymnopus vaccinus & NA & $\mathrm{H} 20$ & NA & NA & {$[8]$} \\
\hline vaccinus & NA & $\mathrm{H} 20$ & A. Gymnopus vaccinus & NA & H15 & NA & NA & [9] \\
\hline velutipes & 314 & $\mathrm{H} 33$ & A. Gymnopus velutipes & (69) & NA & NA & NA & {$[10]$} \\
\hline vialis & NA & $\mathrm{H} 19$ & Gymnop. vialis & $(108)$ & 2:115:85, M49 & 164 & NA & NA \\
\hline Agaricus (Lactifluus) deliciosus & 432 & $\mathrm{H} 17$ & A. Lactifluus deliciosus & NA & 2:138:155, M97 & 209 & NA & NA \\
\hline pallidus & 431 & $\mathrm{H} 46$ & A. Lactifluuspallidus & (68) & 2:143:164, M93 & 209 & NA & NA \\
\hline subdulcis & 433 & H19 & $\begin{array}{l}\text { A. Lactifluus subdulcis } \\
\text { var. minor }\end{array}$ & (3) & $\mathrm{NA}$ & NA & NA & {$[11]$} \\
\hline subdulcis & 433 & $\mathrm{H} 50$ & Lactifl. subdulc. & $(41)$ & NA & 210 & NA & {$[12]$} \\
\hline testaceus & 431 & $\mathrm{H} 47$ & A. Lact. testaceus & NA & NA & 209 & NA & {$[13]$} \\
\hline theiogalus & 431 & $\mathrm{H} 35$ & A. Lactifluus theiogalus & (56) & 2:142:163, M92 & 208 & NA & NA \\
\hline Agaricus (Lepiota) carcharias & 263 & $\mathrm{H} 47$ & A. Lepiota carcharias & NA & $\begin{array}{l}\text { 2:85:13, M10, } \\
\text { H50 }\end{array}$ & 146 & NA & NA \\
\hline carcharias & 263 & $\mathrm{H} 50$ & Lep. carcharias & (30) & $\begin{array}{l}\text { 2:85:13, M10, } \\
\mathrm{H} 47\end{array}$ & 146 & NA & NA \\
\hline caudicinus var. denudatus & 272 & $\mathrm{H} 10$ & Lepiota caudicina $\beta$. & NA & $\mathrm{NA}$ & 150 & NA & {$[14]$} \\
\hline caudicinus var. medius & 272 & $\mathrm{H} 29$ & A. Lepiota caudicina $\gamma$ & NA & NA & 150 & NA & {$[15]$} \\
\hline caudicinus & 271 & H19 & A. Lepiota caudicina & (111) & $2: 87: 16, \mathrm{M} 16$ & 150 & NA & {$[16]$} \\
\hline colubrinus & 258 & $\mathrm{H} 43$ & A. Lepiota colubrinus & 121 & 2:84:11, M8 & 145 & NA & NA \\
\hline squarrosus & 268 & H39 & A. Lepiota squarrosa & (13) & 2:89:20, M12 & 148 & NA & NA \\
\hline Agaricus (Mycena) alliaceus & 375 & $\mathrm{H} 5$ & A. Mycena alliata & NA & 2:123:109, M60 & 190 & NA & NA \\
\hline citrinellus & 384 & $\mathrm{H} 41$ & A. Mycena citrinella & NA & 2:123:107, M60 & 194 & NA & NA \\
\hline [_epiphylla $]$ & NA & $\mathrm{H} 31$ & Mycena epiphylla & NA & NA & NA & NA & {$[17]$} \\
\hline epipterygius & 382 & H14 & A. Mycena epiterygia & NA & $2: 125: 117$, M64 & 193 & NA & NA \\
\hline flavipes & 382 & $\mathrm{H} 3$ & A. Mycena flavipes & NA & $2: 125: 119$, M64 & 193 & NA & NA \\
\hline $\begin{array}{l}\text { praemorsus } \\
\text { galericulatus var. }\end{array}$ & 377 & $\mathrm{H} 29$ & A. Mycena galericulata $\beta$ & NA & 2:123:110, M60 & 191 & NA & {$[18]$} \\
\hline galericulatus & 376 & $\mathrm{H} 38$ & A. Mycena galericulata & NA & $2: 122: 104$, M59 & 191 & NA & [19] \\
\hline galopus & 379 & $\mathrm{H} 25$ & A. Mycena galopus & NA & $2: 121: 102, \mathrm{M} 58$ & 192 & NA & NA \\
\hline haematopus & 379 & $\mathrm{H} 25$ & A. Mycena haematopus & NA & 5:370:292, M61 & 192 & NA & NA \\
\hline leptocephalus & 381 & $\mathrm{H} 20$ & M. leptocephal?? & NA & NA & NA & NA & NA \\
\hline roseus & 393 & $\mathrm{H} 6$ & A. Mycena rosea & NA & 2:124:115, M63 & 197 & NA & NA \\
\hline tenacellus & 387 & $\mathrm{H} 31$ & A.M. tenacella & NA & $\begin{array}{l}\text { 2:125:121, M64, } \\
\text { H35 }\end{array}$ & 195 & NA & NA \\
\hline tenacellus & 387 & $\mathrm{H} 35$ & A. Mycena tenacella? & (57) & $\begin{array}{l}\text { 2:125:121, M64, } \\
\text { H31 }\end{array}$ & 195 & NA & NA \\
\hline tener & 386 & $\mathrm{H} 20$ & A. Mycena tener & NA & 2:122:106, M59 & 194 & NA & NA \\
\hline Agaricus (Omphalia) amethysteus & 465 & $\mathrm{H} 36$ & $\begin{array}{l}\text { A. Omphalia } \\
\text { amethystenus }\end{array}$ & 74 & 2:153:186, M89. & 222 & NA & {$[20]$} \\
\hline bellus & 452 & $\mathrm{H} 27$ & A. Omphal. Bella & (79) & 2:156:195, M102 & 218 & NA & NA \\
\hline campanella & 469 & $\mathrm{H} 4$ & $\begin{array}{l}\text { A. Omphalia } \\
\text { campanella }\end{array}$ & NA & $\begin{array}{l}\text { 2:160:204, } \\
\text { M100, H25 }\end{array}$ & 224 & NA & NA \\
\hline
\end{tabular}


Table 2. (Continued).

\begin{tabular}{|c|c|c|c|c|c|c|c|c|}
\hline Standardized name & Pers. 1801 & Plate & Verb. name & Verb. no. & Other figures & Conspectus & $\begin{array}{l}\text { Verb. } \\
\text { notes }\end{array}$ & Comments \\
\hline campanella & 469 & $\mathrm{H} 25$ & $\begin{array}{l}\text { A. Omphalia } \\
\text { campanella }\end{array}$ & NA & $\begin{array}{l}\text { 2:160:204, } \\
\text { M100, H4 }\end{array}$ & 224 & NA & NA \\
\hline candicans & 456 & $\mathrm{H} 43$ & A. Omphalia candicans & NA & 2:154:190, M103 & 219 & NA & NA \\
\hline epichysium & 462 & $\mathrm{H} 18$ & A. Omphalia epichysium & 66 & $2: 155: 193$ & 222 & NA & {$[21]$} \\
\hline _epichysium & NA & $\mathrm{H} 29$ & $\begin{array}{l}\text { A. Omphalia epichysium } \\
\alpha\end{array}$ & NA & NA & 222 & NA & {$[22]$} \\
\hline epiphyllus & 468 & $\mathrm{H} 1$ & $\begin{array}{l}\text { A. Gymnopus ramealis. } \\
\text { Omph. Epiphylla }\end{array}$ & NA & $?$ & 223 & NA & {$[23]$} \\
\hline ericetorum & 472 & $\mathrm{H} 45$ & A. Omph. ericetorum & $(46)$ & $\begin{array}{l}\text { 2:160:206, } \\
\text { M100. }\end{array}$ & 225 & NA & {$[24]$} \\
\hline farinaceus & 453 & $\mathrm{H} 38$ & A. Om. farinacea & NA & 2:153:185, M89. & 219 & NA & NA \\
\hline gibbus & 449 & $\mathrm{H} 36$ & A. Om. Gibba & $\mathrm{NA}$ & $\begin{array}{l}\text { 2:154:188, } \\
\text { M103, H33 }\end{array}$ & 217 & NA & NA \\
\hline gibbus & 449 & $\mathrm{H} 33$ & A. Omphalia gibba & $(31)$ & $\begin{array}{l}\text { 2:154:188, } \\
\text { M103, H36 }\end{array}$ & 217 & NA & NA \\
\hline gilvus & 448 & $\mathrm{H} 45$ & A. Omphalia gilva & (86) & 2:157:196, M104 & 216 & NA & NA \\
\hline nigrella & 463 & H15 & A. Omphalia nigrella & NA & $\begin{array}{l}2: 153: 187, \text { M89, } \\
\text { H50 }\end{array}$ & 222 & NA & NA \\
\hline nigrella & 463 & $\mathrm{H} 50$ & A. Omphalia nigrella & (9) & $\begin{array}{l}\text { 2:153:187, M89, } \\
\text { H15 }\end{array}$ & 222 & NA & {$[25]$} \\
\hline prunulus & 457 & $\mathrm{H} 31$ & A. Omphalia prunula & NA & 2:157:197, M104 & 220 & NA & NA \\
\hline tigrinus & 458 & $\mathrm{H} 44$ & A. Omph. tigrinus & $(5)$ & NA & NA & NA & NA \\
\hline Agaricus (Pleuropus) epigaeus & 484 & $\mathrm{H} 14$ & A. Pleuropus epigaeus & NA & $5: 376: 308$ & 232 & NA & NA \\
\hline ostreatus & 477 & H12 & A. Pleuropus ostreatus & NA & $\begin{array}{l}\text { 2:163:213, } \\
\mathrm{M} 107, \mathrm{H} 33\end{array}$ & 228 & NA & NA \\
\hline ostreatus & 477 & $\mathrm{H} 33$ & A. Pleuropus ostreatus & (58) & $\begin{array}{l}\text { 2:163:213, } \\
\text { M107, H12 }\end{array}$ & 228 & NA & NA \\
\hline tessellatus & 474 & $\mathrm{H} 49$ & A. Pleur. tessellatus & NA & 2:162:210 & 226 & NA & NA \\
\hline variabilis & 483 & H16 & A. Pleuropus variabilis & NA & $2: 165: 217$, M110 & 231 & "Stat. jun." & $\mathrm{NA}$ \\
\hline Agaricus (Pratella) campestris & 418 & $\mathrm{H} 2$ & A. Pratella campestris & NA & $2: 132: 142, \mathrm{M} 71$ & 204 & NA & NA \\
\hline echinatus & 419 & $\mathrm{H} 43$ & A. Pratella echinata & $(76)$ & 2:134:147 & 204 & NA & NA \\
\hline edulis & 418 & H12 & Pratella edulis $\beta$ & NA & NA & NA & NA & {$[26]$} \\
\hline fuligineus & 427 & $\mathrm{H} 23$ & A. Prat. fuliginea & $(10)$ & 2:133:145, M72 & 207 & NA & NA \\
\hline montanus & 428 & $\mathrm{H} 29$ & A. Pratella montana & NA & 2:137:153, M96 & 207 & NA & $\mathrm{NA}$ \\
\hline _ pascuus & 427 & $\mathrm{H} 28$ & A. Pratella pascua & NA & $\begin{array}{l}\text { 2:133:144, M72, } \\
\text { H3 }\end{array}$ & 207 & NA & NA \\
\hline _ pascuus & 427 & $\mathrm{H} 3$ & A. Prat.pascua & NA & $\begin{array}{l}\text { 2:133:144, M72, } \\
\text { H28 }\end{array}$ & 207 & NA & {$[27]$} \\
\hline praecox & 420 & $\mathrm{H} 33$ & A. Prat.praecox & 104 & $\begin{array}{l}\text { 2:135:149, M73, } \\
\text { H38 }\end{array}$ & 205 & NA & NA \\
\hline praecox & 420 & $\mathrm{H} 38$ & A. Pratella praecox & NA & $\begin{array}{l}2: 135: 149, \text { M73, } \\
\text { H33 }\end{array}$ & 205 & NA & NA \\
\hline Agaricus (Russula) furcatus & 446 & $\mathrm{H} 24$ & A. Russula furcata & NA & 2:145:170, M82 & 215 & NA & NA \\
\hline ochroleuca & 443 & $\mathrm{H} 25$ & A. Russula ochroleuca & NA & 2:150:181, M85 & 213 & NA & NA \\
\hline Amanita citrina & 251 & $\mathrm{H} 9$ & Amanita citrina & $(11)$ & 2:81:7, M4 & 143 & NA & NA \\
\hline livida & 247 & $\mathrm{H} 1$ & Amanita livida & NA & $2: 80: 4, \mathrm{M} 3$ & 141 & NA & NA \\
\hline spadicea & 248 & $\mathrm{H} 4$ & Amanita spadicea & NA & 2:81:6, M4 & 141 & NA & NA \\
\hline umbrina & 254 & $\mathrm{H} 26$ & Amanita umbrina & NA & 2:79:3, M2 & 143 & NA & NA \\
\hline Arcyria cinerea & 184 & $\mathrm{H} 30$ & Arcyria cinerea & NA & $1: 57: 02$ & 101 & NA & NA \\
\hline flava & 184 & $\mathrm{H} 30$ & Arcyria flava & NA & 1:57:01 & 101 & NA & NA \\
\hline _ punicea & 185 & $\mathrm{H} 4$ & Arcyria punicea & NA & 1:57:04 & 101 & NA & NA \\
\hline Boletus citrinus & 524 & H13 & Boletus citrinus obsolet. & NA & 3:185:20, M131 & 246 & NA & NA \\
\hline
\end{tabular}


Table 2. (Continued).

\begin{tabular}{|c|c|c|c|c|c|c|c|c|}
\hline Standardized name & Pers. 1801 & Plate & Verb. name & Verb. no. & Other figures & Conspectus & $\begin{array}{l}\text { Verb. } \\
\text { notes }\end{array}$ & Comments \\
\hline fuligineus & 516 & H14 & Boletus fuligineus & NA & NA & NA & NA & NA \\
\hline leptocephalus & 519 & $\mathrm{H} 23$ & Boletus leptocephalus & NA & $\mathrm{NA}$ & $\mathrm{NA}$ & NA & NA \\
\hline Boletus (Suillus) annulatus & 503 & $\mathrm{H} 9$ & Boletus annulatus & $\mathrm{NA}$ & 3:176:1, M113 & 238 & NA & NA \\
\hline circinans & 505 & $\mathrm{H} 18$ & Boletus circinans & NA & 5:382:50, M121 & 239 & NA & NA \\
\hline scaber & 505 & $\mathrm{H} 21$ & Boletus scaber & NA & 3:178:5, M118 & 239 & NA & NA \\
\hline Bovista nigrescens & 136 & $\mathrm{H} 10$ & Bovista nigrescens & NA & $1: 42: 01$ & 79 & NA & NA \\
\hline Ceratium porioides Alb. \& Schwein. & NA & $\mathrm{H} 21$ & Poria cornuta Nobis & NA & $\begin{array}{l}\text { 5:429:2, M286, } \\
\text { C2:6 }\end{array}$ & 359 & NA & {$[28]$} \\
\hline Clavaria pistillaris & 597 & $\mathrm{H} 20$ & Clavaria pistillaris & NA & 5:407:24, M206 & 289 & NA & NA \\
\hline Clavaria (Ramaria) abietina & 588 & $\mathrm{H} 36$ & Clavaria abietina & $\mathrm{NA}$ & 3:222:4, M198 & 286 & $\mathrm{NA}$ & NA \\
\hline cristata & 591 & H19 & Clavaria cristata & NA & 3:224:9, M202 & 287 & NA & NA \\
\hline pratensis & 590 & $\mathrm{H} 48$ & Clavaria pratensis & NA & 3:222:5, M198 & 287 & NA & NA \\
\hline Clavaria [monstrosa] & $\mathrm{NA}$ & $\mathrm{H} 20$ & Clavaria monstrosa & NA & NA & NA & NA & NA \\
\hline [monstrosa] & $\mathrm{NA}$ & $\mathrm{H} 30$ & Clavaria monstrosa & $\mathrm{NA}$ & $\mathrm{NA}$ & NA & NA & NA \\
\hline sp. & NA & $\mathrm{H} 7$ & Clavaria & NA & NA & NA & NA & NA \\
\hline sp. & NA & $\mathrm{H} 41$ & Clavaria & NA & NA & NA & NA & NA \\
\hline Cribraria argillacea & 193 & $\mathrm{H} 30$ & Cribraria argillacea & NA & $4: 329: 8$ & 106 & NA & NA \\
\hline cernua & 189 & $\mathrm{H} 36$ & Cribraria cernua & NA & $4: 328: 3$ & 104 & NA & NA \\
\hline Cyathus scutellatus & 239 & $\mathrm{H} 43$ & Cyathus scutellatus & NA & $1: 77: 1$ & 139 & NA & NA \\
\hline striatus & 237 & $\mathrm{H} 8$ & Cyathus striatus & $\mathrm{NA}$ & $1: 76: 1$ & 138 & NA & NA \\
\hline Diderma contortum & 166 & $\mathrm{H} 28$ & Diderma contortum & NA & NA & NA & NA & NA \\
\hline vernicosum & 165 & $\mathrm{H} 23$ & Diderma vernicosum & NA & $1: 52: 02$ & 89 & $\mathrm{NA}$ & NA \\
\hline Fuligo rufa & 159 & $\mathrm{H} 37$ & Fuligo rufa & $\mathrm{NA}$ & $4: 318: 1$ & 86 & NA & NA \\
\hline vaporaria & 161 & $\mathrm{H} 18$ & Fuligo vaporaria & NA & $4: 319: 5$ & 86 & NA & NA \\
\hline Helotium galeatum & 678 & $\mathrm{H} 8$ & Helotium galeatum & NA & 3:268:3, M239 & 350 & NA & NA \\
\hline Himantia candida & 704 & $\mathrm{H} 35$ & Himantia candida & NA & 5:449:2, M276 & 373 & NA & NA \\
\hline Leotia circinans & 612 & $\mathrm{H} 4$ & Leotia circinans & 4 & $3: 230: 4$, M224 & 297 & NA & NA \\
\hline lubrica & 613 & H15 & Leotia lubrica & NA & 3:230:3, M224 & 298 & NA & NA \\
\hline mitrula & 611 & H35 & Leotia mitrula & $\mathrm{NA}$ & 3:229:2, M223 & 295 & NA & NA \\
\hline Licea variabilis & 197 & $\mathrm{H} 38$ & Licea variabilis & $\mathrm{NA}$ & NA & NA & NA & NA \\
\hline Merisma foetidum & 584 & H17 & Merisma foetidum & $\mathrm{NA}$ & 3:220:1, M196 & 284 & $\begin{array}{l}\text { "Spec. } \\
\text { parv." }\end{array}$ & NA \\
\hline Merulius (Cantharellus) aurantiacus & 488 & $\mathrm{H} 41$ & Merulius aurantiacus & $(59)$ & 2:170:230, M155 & 233 & NA & NA \\
\hline umbonatus & 491 & $\mathrm{H} 50$ & Merulius umbonatus & $(42)$ & 5:379:314, M157 & 235 & NA & NA \\
\hline Merulius crispus & 495 & $\mathrm{H} 42$ & Merulius crispus & NA & $\begin{array}{l}\text { 2:172:233, } \\
\text { M158, H49 }\end{array}$ & 236 & NA & [29] \\
\hline crispus & 495 & H49 & Merul. crisp. inexplicat. & $\mathrm{NA}$ & $\mathrm{NA}$ & NA & NA & {$[30]$} \\
\hline Mesenterica grisea Alb. \& Schwein. & NA & H11 & $\begin{array}{l}\text { Mesenteriaca grisea } \\
\text { Nob. }\end{array}$ & NA & 5:452:2, M279 & 374 & NA & NA \\
\hline Monilia candida & 692 & $\mathrm{H} 41$ & Monilia candida. glauca & $\mathrm{NA}$ & 5:436:6, M281 & 364 & NA & {$[31]$} \\
\hline Mucor mucedo & 201 & H11 & Mucor mucedo & NA & $1: 62: 3$ & 111 & $\mathrm{NA}$ & NA \\
\hline Naemaspora crocea & 109 & $\mathrm{H} 7$ & Naemaspora crocea & NA & $1: 34: 03$ & 67 & NA & {$[32]$} \\
\hline crocea $[$ monstrosa $]$ & 109 & $\mathrm{H} 27$ & $\begin{array}{l}\text { Naemaspora crocea. } \\
\text { monstros. }\end{array}$ & NA & NA & NA & NA & {$[33]$} \\
\hline Peziza aeruginosa & 663 & $\mathrm{H} 23$ & Peziza aeruginosa & $\mathrm{NA}$ & 3:256:60, M261 & 334 & NA & NA \\
\hline _alutacea & 638 & $\mathrm{H} 12$ & Peziza alutacea & NA & 5:417:101, M229 & 310 & NA & NA \\
\hline$\ldots$ anomala & 656 & $\mathrm{H} 42$ & Peziza anomala & NA & $3: 248: 37, \mathrm{M} 253$ & 326 & NA & NA \\
\hline _aurantia & 637 & $\mathrm{H} 16$ & Peziza aurantia & NA & 5:416:99, M228 & 310 & NA & NA \\
\hline bolaris & 658 & $\mathrm{H} 35$ & Peziza bolaris & NA & 3:253:50, M258 & 330 & NA & NA \\
\hline
\end{tabular}


Table 2. (Continued).

\begin{tabular}{|c|c|c|c|c|c|c|c|c|}
\hline Standardized name & Pers. 1801 & Plate & Verb. name & Verb. no. & Other figures & Conspectus & $\begin{array}{l}\text { Verb. } \\
\text { notes }\end{array}$ & Comments \\
\hline cinerea & 634 & $\mathrm{H} 21$ & Pezizacinerea & NA & 3:239:5, M244 & 307 & NA & NA \\
\hline crenata & 647 & $\mathrm{H} 2$ & Peziza crena[ta] & NA & 5:420:110, M232 & 314 & NA & NA \\
\hline cyathoidea var. tenella & 662 & $\mathrm{H} 13$ & Peziza cyathoidea $\beta$ & NA & NA & NA & NA & NA \\
\hline fructigena & 660 & $\mathrm{H} 30$ & Peziza fructigena & NA & 3:254:53, M259 & 331 & NA & NA \\
\hline hemisphaerica & 647 & $\mathrm{H} 41$ & Peziza hemisphaerica & NA & 3:244:17, M248 & 316 & NA & NA \\
\hline leporina & 637 & $\mathrm{H} 3$ & Peziza leporina & NA & $3: 241: 11, \mathrm{M} 246$ & 309 & NA & NA \\
\hline Leucoloma & 665 & $\mathrm{H} 2$ & Peziza leucoloma & NA & $3: 258: 68, \mathrm{M} 263$ & 335 & NA & NA \\
\hline macropus var. villosa & 645 & $\mathrm{H} 16$ & Peziza macropus $\beta$ & NA & 5:418:104 & NA & NA & [34] \\
\hline nivea & 653 & $\mathrm{H} 5$ & Peziza nivea & NA & $\mathrm{NA}$ & NA & $\mathrm{NA}$ & [35] \\
\hline r r rizopus Alb. \& Schwein. & NA & $\mathrm{H} 28$ & $\begin{array}{l}\text { Peziza rbizophora } \\
\text { Nobis }\end{array}$ & NA & $\begin{array}{l}\text { 3:250:42, M255, } \\
\text { C1:4 }\end{array}$ & 317 & NA & NA \\
\hline sarcoides & 633 & $\mathrm{H} 24$ & Peziza sarcoidea & NA & 3:238:3, M243 & 305 & NA & NA \\
\hline scutellata & 650 & $\mathrm{H} 13$ & Peziza scutellata & NA & $3: 245: 21, \mathrm{M} 250$ & 320 & NA & NA \\
\hline stercoraria & 676 & $\mathrm{H} 6$ & Peziza stercoraria & NA & $3: 245: 22, \mathrm{M} 250$ & 320 & NA & {$[36]$} \\
\hline sulphurea & 649 & H19 & Peziza sulphurea & NA & 3:244:19, M249 & 319 & NA & NA \\
\hline umbrina & 638 & $\mathrm{H} 16$ & Peziza umbrina & NA & 5:417:102, M229 & 310 & NA & NA \\
\hline (Solenia) incana & 675 & $\mathrm{H} 7$ & Peziza Solenia incana & NA & $3: 266: 96, \mathrm{M} 271$ & 346 & NA & NA \\
\hline sp. & NA & $\mathrm{H} 6$ & Peziza & NA & NA & NA & NA & NA \\
\hline sp. & NA & $\mathrm{H} 20$ & Peziza & NA & NA & NA & NA & NA \\
\hline sp. & NA & $\mathrm{H} 40$ & Peziza & NA & NA & NA & NA & NA \\
\hline sp. & NA & $\mathrm{H} 41$ & Peziza & NA & NA & NA & NA & $\mathrm{NA}$ \\
\hline Physarum confluens & 169 & $\mathrm{H} 26$ & Physarum confluens. & NA & 4:322:10 & 91 & NA & NA \\
\hline farinaceum & 174 & H11 & Physarum farinaceum & NA & $\begin{array}{l}\text { 1:54:4, H21, } \\
\mathrm{H} 28, \mathrm{H} 42\end{array}$ & 96 & NA & NA \\
\hline farinaceum & 174 & $\mathrm{H} 21$ & Physarum farinaceum & NA & $\begin{array}{l}\text { 1:54:4, H11, } \\
\text { H28, H42 }\end{array}$ & 96 & NA & NA \\
\hline farinaceum & 174 & $\mathrm{H} 28$ & Physarum farinaceum & NA & $\begin{array}{l}\text { 1:54:4, H11, } \\
\text { H21, H42 }\end{array}$ & 96 & NA & NA \\
\hline farinaceum & 174 & $\mathrm{H} 42$ & Physarum farinaceum & NA & $\begin{array}{l}\text { 1:54:4, H11, } \\
\mathrm{H} 21, \mathrm{H} 28\end{array}$ & 96 & NA & NA \\
\hline squamulosum & 174 & $\mathrm{H} 16$ & Physarum squamulosum & NA & $1: 54: 02$ & 95 & NA & NA \\
\hline Puccinia Rosae & 215 & $\mathrm{H} 18$ & Puccinia Rosae & NA & NA & NA & NA & {$[37]$} \\
\hline Sphaeria fimbriata & 36 & $\mathrm{H} 42$ & Sphaeria fimbriata & NA & 1:13:01 & 17 & NA & NA \\
\hline fusca & 12 & $\mathrm{H} 37$ & Sphaeria fusca & NA & 1:04:02 & 4 & $\mathrm{NA}$ & $\mathrm{NA}$ \\
\hline hypoxylon & 5 & $\mathrm{H} 3$ & Sphaeria hypoxylon & NA & 1:02:01 & 2 & NA & NA \\
\hline lagenaria & 58 & H11 & Sphaeria lagenaria & NA & 1:19:03 & 28 & NA & NA \\
\hline moriformis & 86 & $\mathrm{H} 37$ & Sphaeria moriformis & NA & $1: 24: 02$ & 43 & $\mathrm{NA}$ & $\mathrm{NA}$ \\
\hline ovina & 71 & $\mathrm{H} 6$ & Sphaeria ovina & NA & $1: 22: 03$ & 36 & NA & NA \\
\hline pustulata & 41 & $\mathrm{H} 32$ & Sphaeria pustulata & NA & 4:284:103 & 21 & NA & NA \\
\hline quaternata & 45 & H6 & Sphaeria quaternata & NA & $4: 285: 104$ & 23 & NA & NA \\
\hline rubiformis & 9 & $\mathrm{H} 37$ & Sphaeria rubiformis & NA & $1: 03: 02$ & 3 & NA & NA \\
\hline sp. & NA & $\mathrm{H} 44$ & Sphaeria? & NA & NA & NA & NA & NA \\
\hline stigma & 21 & $\mathrm{H} 5$ & Sphaeria stigma & NA & 1:6:2, H37 & 9 & NA & NA \\
\hline stigma & 21 & $\mathrm{H} 37$ & Sphaeria stigma & NA & $1: 6: 2, \mathrm{H} 5$ & 9 & NA & NA \\
\hline subulata & 94 & $\mathrm{H} 4$ & Sphaeria subulata & NA & $1: 25: 04$ & 52 & NA & NA \\
\hline spp. [5 illustrations] & NA & $\mathrm{H} 34$ & $\begin{array}{l}\text { Sphaeriae quaedam } \\
\text { indistinctae. }\end{array}$ & NA & NA & NA & NA & $\mathrm{NA}$ \\
\hline Sphaerobolus stellatus & 115 & H19 & Sphaerobolus stellatus & NA & $1: 36: 01$ & 70 & NA & NA \\
\hline Stemonitis fasciculata & 187 & $\mathrm{H} 30$ & Stemonitis fasciculata & NA & $1: 58: 03$ & 102 & NA & NA \\
\hline
\end{tabular}


Table 2. (Continued).

\begin{tabular}{|c|c|c|c|c|c|c|c|c|}
\hline Standardized name & Pers. 1801 & Plate & Verb. name & Verb. no. & Other figures & Conspectus & $\begin{array}{l}\text { Verb. } \\
\text { notes }\end{array}$ & Comments \\
\hline ovata & 189 & $\mathrm{H} 21$ & Stemonitis ovata & NA & $1: 58: 04$ & 104 & NA & {$[38]$} \\
\hline ovata var. nigra & 189 & $\mathrm{H} 40$ & Stemonitis ovata $\beta$ & $\mathrm{NA}$ & NA & NA & NA & [39] \\
\hline typhina & 187 & $\mathrm{H} 7$ & Stemonits typhina & $\mathrm{NA}$ & 1:58:02 & 102 & NA & NA \\
\hline sp. & NA & H16 & Stemonitis & NA & NA & NA & NA & NA \\
\hline Thelephora (Corticium) hydnoidea & 576 & $\mathrm{H} 18$ & Thelephora C. hydnoidea & NA & 3:215:20, M186 & 279 & NA & NA \\
\hline Thelephora (Stereum) rugosa & 569 & $\mathrm{H} 32$ & Thelephora S. rugosa & NA & 3:210:6, M181 & 274 & NA & NA \\
\hline Tremella dubia & 630 & $\mathrm{H} 8$ & Tremella dubia & $\mathrm{NA}$ & $3: 237: 13, \mathrm{M} 213$ & 305 & NA & NA \\
\hline encephala & 623 & $\mathrm{H} 42$ & Tremella encephala & $\mathrm{NA}$ & 3:233:3, M210 & 301 & NA & NA \\
\hline spiculosa & 624 & H44 & Tremella spiculosa & $\mathrm{NA}$ & $3: 235: 7$, M212 & 302 & NA & NA \\
\hline undulata & 626 & $\mathrm{H} 1$ & Tremella undulata & $\mathrm{NA}$ & NA & $\mathrm{NA}$ & NA & NA \\
\hline Trichia nigripes & 178 & $\mathrm{H} 6$ & Trichia nigripes & NA & $1: 55: 04$ & 99 & NA & NA \\
\hline nitens & 180 & $\mathrm{H} 36$ & Trichia nitens & $\mathrm{NA}$ & 1:56:03 & 99 & NA & NA \\
\hline olivacea & 180 & H11 & Trichia olivacea & NA & $1: 56: 02$ & 99 & $\mathrm{NA}$ & NA \\
\hline rubiformis & 176 & $\mathrm{H} 6$ & Trichia rubiformis & NA & 1:55:02 & 98 & "statu jun." & NA \\
\hline varia & 181 & $\mathrm{H} 28$ & Trichia varia & $\mathrm{NA}$ & $1: 56: 04$ & 100 & NA & NA \\
\hline Trichoderma tuberculatum & 234 & $\mathrm{H} 32$ & Trichoderma tuberculat. & $\mathrm{NA}$ & 4:348:7, H40 & 136 & NA & NA \\
\hline tuberculatum & 234 & $\mathrm{H} 40$ & Trichoderma tuberculat. & $\mathrm{NA}$ & 4:348:7, H32 & 136 & NA & NA \\
\hline Tuber album & 128 & $\mathrm{H} 8$ & Tuber album & $\mathrm{NA}$ & $4: 309: 2$ & 77 & NA & NA \\
\hline Tubercularia vulgaris & 112 & $\mathrm{H} 49$ & Tubercularia vulgaris & $\mathrm{NA}$ & $1: 35: 01$ & 69 & NA & NA \\
\hline Tubulina fragiformis & 198 & $\mathrm{H} 4$ & Tubulina fragiformis & $\mathrm{NA}$ & $1: 61: 1$ & 110 & NA & NA \\
\hline Uredo rosae centifoliae & 215 & H18 & Uredo rosae centifoliae & $\mathrm{NA}$ & $4: 339: 16$ & 123 & $\begin{array}{l}\text { "cum } \\
\text { Puccinia } \\
\text { Rosae" }\end{array}$ & NA \\
\hline White cup fungus on wood & $\mathrm{NA}$ & H1 & $\mathrm{NA}$ & NA & $\mathrm{NA}$ & NA & NA & NA \\
\hline Pyrenomycete on wood & $\mathrm{NA}$ & $\mathrm{H} 5$ & $\mathrm{NA}$ & $\mathrm{NA}$ & NA & NA & NA & NA \\
\hline Tiny orange cup fungus & $\mathrm{NA}$ & $\mathrm{H} 12$ & $\mathrm{NA}$ & NA & NA & NA & NA & NA \\
\hline Tiny nervicolous cup fungus & $\mathrm{NA}$ & $\mathrm{H} 12$ & $\mathrm{NA}$ & NA & NA & NA & $\mathrm{NA}$ & NA \\
\hline Myxomycete? & $\mathrm{NA}$ & $\mathrm{H} 13$ & $\mathrm{NA}$ & $\mathrm{NA}$ & $\mathrm{NA}$ & NA & $\mathrm{NA}$ & NA \\
\hline White ramarioid fungus & $\mathrm{NA}$ & H15 & NA & $\mathrm{NA}$ & $\mathrm{NA}$ & $\mathrm{NA}$ & NA & NA \\
\hline Tiny bolete with reticulate stipe & NA & $\mathrm{H} 17$ & NA & NA & NA & NA & NA & NA \\
\hline Confluent fungi on wood & NA & $\mathrm{H} 17$ & NA & NA & NA & NA & NA & NA \\
\hline Leaf parasite & NA & $\mathrm{H} 21$ & NA & NA & $\mathrm{NA}$ & $\mathrm{NA}$ & NA & NA \\
\hline Agaric & $\mathrm{NA}$ & $\mathrm{H} 22$ & $\mathrm{NA}$ & $(53)$ & $\mathrm{NA}$ & $\mathrm{NA}$ & NA & NA \\
\hline Stipitate fungus & $\mathrm{NA}$ & $\mathrm{H} 24$ & $\mathrm{NA}$ & NA & $\mathrm{NA}$ & NA & NA & NA \\
\hline Agaric & $\mathrm{NA}$ & $\mathrm{H} 24$ & $\mathrm{NA}$ & $\mathrm{NA}$ & $\mathrm{NA}$ & NA & NA & NA \\
\hline Yellow jelly fungus? & $\mathrm{NA}$ & $\mathrm{H} 25$ & $\mathrm{NA}$ & NA & $\mathrm{NA}$ & NA & $\mathrm{NA}$ & NA \\
\hline Pink fungoid pustules on wood & NA & $\mathrm{H} 26$ & NA & NA & NA & NA & NA & NA \\
\hline Gray agaric & $\mathrm{NA}$ & $\mathrm{H} 27$ & NA & $(55)$ & $\mathrm{NA}$ & NA & NA & NA \\
\hline Fungal growth? & $\mathrm{NA}$ & $\mathrm{H} 28$ & Inexplicatus Fungus & NA & $\mathrm{NA}$ & NA & NA & NA \\
\hline Anamorphic fungus & NA & $\mathrm{H} 28$ & NA & NA & NA & NA & NA & NA \\
\hline White tubular fungus on wood & $\mathrm{NA}$ & $\mathrm{H} 30$ & $\mathrm{NA}$ & NA & $\mathrm{NA}$ & NA & NA & NA \\
\hline Small gray umbonate agaric & $\mathrm{NA}$ & $\mathrm{H} 31$ & $\mathrm{NA}$ & $\mathrm{NA}$ & $\mathrm{NA}$ & NA & NA & NA \\
\hline Black perithecioid fungus on wood & $\mathrm{NA}$ & $\mathrm{H} 32$ & NA & $\mathrm{NA}$ & $\mathrm{NA}$ & NA & NA & NA \\
\hline Black ramarioid fungus & $\mathrm{NA}$ & $\mathrm{H} 45$ & $\mathrm{NA}$ & $\mathrm{NA}$ & $\mathrm{NA}$ & NA & NA & NA \\
\hline Gray agaric with narrow stipe & $\mathrm{NA}$ & $\mathrm{H} 45$ & $\mathrm{NA}$ & NA & $\mathrm{NA}$ & NA & NA & NA \\
\hline Myxomycete on wood? & $\mathrm{NA}$ & $\mathrm{H} 45$ & $\mathrm{NA}$ & NA & $\mathrm{NA}$ & NA & NA & NA \\
\hline Stipitate cup fungus on wood & NA & $\mathrm{H} 46$ & NA & NA & NA & NA & NA & NA \\
\hline Tan agaric with decurrent gills & NA & $\mathrm{H} 47$ & NA & NA & NA & NA & NA & NA \\
\hline Yellow agaric with narrow stipe & $\mathrm{NA}$ & $\mathrm{H} 47$ & $\mathrm{NA}$ & $(48)$ & $\mathrm{NA}$ & NA & NA & {$[40]$} \\
\hline
\end{tabular}


Table 2. (Continued).

\begin{tabular}{|l|l|l|l|l|l|l|l|l|}
\hline Standardized name & Pers. 1801 & Plate & Verb. name & Verb. no. & Other figures & Conspectus & $\begin{array}{l}\text { Verb. } \\
\text { notes }\end{array}$ & Comments \\
\hline Crust fungus on wood & NA & H49 & NA & NA & NA & NA & NA & NA \\
\hline Fungal growth? & NA & H49 & Substantia aquatica & NA & NA & NA & NA & NA \\
\hline
\end{tabular}

[1] Cfr. A. (Cortinaria) bicolor (Conspectus, p. 154), figs. 2:91:24 and M23. These figs. were originally labeled A. nudus, however, in Vol. 2, Index A, nuda is struck and bicolor is inserted.

[2] 2:117:90 copied from H46 (Fig. 3B).

[3] Cfr. A. (G.) humilis in Persoon (1801), p. 360 and in the Conspectus, p. 180. In Vol. 2, Index A, humilis is entered above $A$. (G.) comitialis, a taxon that is not listed in the Conspectus, though these figs. are labeled by this name. However, in H48, humilis is struck and comitialis is inserted.

[4] 2:116:89 copied from H7 (Fig. 3A).

[5] Cfr. A. (Gymnopus) parasiticus (Conspectus, p. 187), figs. 2:117:92 and M52. In the indices for Vol. 2, lycoperdoides is struck and parasiticus is inserted.

[6] Fig. 2:113:77 is a partial copy of $\mathrm{H} 23$ (Fig. 3E).

[7] Fig. 2:104:56 copied from H47 (Fig. 3C).

[8] Cfr. A. (Cortinaria) vaccina (Conspectus p. 159), figs. 2:91:25 and M23.

[9] See footnote 8 .

[10] In this fig. label, velutipes is struck but no other name is given. Cfr. Conspectus p. 165 and figs. 2:115:84, M49.

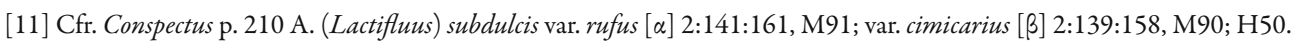

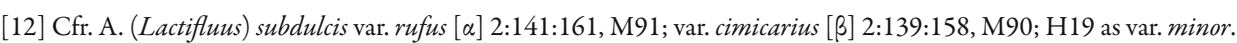

[13] Cfr. 2:138:154 as $\alpha$ and 2:139:156 as $\beta$.

[14] Cfr. A. (Lepiota) caudicinus 2:87:16, M16, H19 and var. medius $[\gamma] \mathrm{H} 29$.

[15] Cfr. A. (Lepiota) caudicinus 2:87:16, M16, H19 and var. denudatus [ $\beta]$ H10.

[16] Cfr. A. (Lepiota) caudicinus var. denudatus [ $\beta] \mathrm{H} 10$ and var. medius $[\gamma] \mathrm{H} 29$.

[17] Cfr. A. (Omphalia) epiphyllus.

[18] In Conspectus, the $\beta$ var. is albida. Cfr. A. (Mycena) galericulatus 2:122:104, M59, H38.

[19] Cfr. A. (Mycena) galericulata var. praemorsus 2:123:110, M60, H29.

[20] farinacea is struck and amethystenus inserted. Cfr. A. (Omphalia) farinaceus 2:153:185, M89, H38.

[21] Cfr. A. (Omphalia) epichysium var. communis [a] H29.

[22] A. (Omphalia) epichysium var. communis Alb. \& Schwein. Cfr. A. (Omphalia) epichysium 2:155:193, H18.

[23] Cfr. H1 with A. (Omphalia) epiphylla: 2:161:209 (M101 fig. in lower center is a copy of this) and with A. (Omphalia) epiphylla var. $\alpha$ (var. vulgaris in the Conspectus): 2:161:207 (M101 fig. on upper left is a copy of this). Cfr. A. (Mycena) epiphylla: H31. Figs. for A. (Gymnopus) ramealis are given with the listing for that taxon.

[24] Cfr. A. (Omphalia) ericetorum var. vaillantii [ß]: 2:161:206b, M101 (fig. on upper right).

[25] Fig. 2:153:187 copied from H50 (Fig. 3D).

[26] Cfr. Conspectus p. 203 A. (Pratella) edulis, figs. 2:132:141, M71.

[27] "A. Pr. pascua var. $\beta$ " in faint pencil.

[28] Ceratium porioides Alb. \& Schwein. C2:6 labeled “Certatium porioides $\beta$, flavum” in Explicatio iconum, p. xviii.

[29] Cfr. Merulius crispus inexplicat. H49.

[30] Cfr. Merulius crispus 2:172:233, M158, H42.

[31] Cfr. Monilia glauca 5:436:4, M281.

[32] Cfr. Naemaspora crocea. monstros. H27

[33] Cfr. Naemaspora crocea 1:34:3, H7.

[34] Cfr. Peziza macropus 5:418:104, M230.

[35] In Pers. 1801 as P. virginiana. Cfr. Peziza nivea in Sowerby [1795-] 1797 Tab. 65. The fungus depicted in H5 is black.

[36] This taxon in Pers. 1801 as synonym of Ascobolus furfuraceus.

[37] See Uredo rosae centifoliae.

[38] Cfr. Stemonitis ovata var. nigra [ $\beta] \mathrm{H} 40$.

[39] Cfr. Stemonitis ovata 1:58:4, H21.

[40] "Cort. decora" (in pencil). 
(Torrens 2005), and did not see Schweinitz again until sixteen years later at a reunion in Bethlehem (PA), shortly prior to January 1818 (Steinhauer 1818). Daniel Steinhauer, after learning of his brother's declining health by consumption and a series of family tragedies, emigrated to America, arriving in February 1818. The brothers were reunited only briefly, however, as H. Steinhauer died in July at the age of 36 (Torrens 2005).

The circumstances of how the Harvard Icones came into D. Steinhauer's or Darlington's hands, or with what understanding with Schweinitz was in place for the volume to be made a gift, are currently unknown. It is possible that in 1826 the Harvard Icones was in D. Steinhauer's possession. Henry Steinhauer, along with the topographical engineer and botanist, Major John Eatton Leconte (1784-1860) had endeavored since 1818, with Schweinitz's assistance, to have copies made of figures from Schweinitz's fivevolume set. Henry Steinhauer notes in an 1818 letter to Leconte that Schweinitz, during their reunion, had left him with his "Icones Fungorum Niesk'm" in order to begin the process of getting them copied (Steinhauer 1818). These efforts resulted in the Michigan Icones that comprises copies of illustrations from volumes 2, 3, and 5 . We may speculate that if the Harvard Icones was somehow in H. Steinhauer's possession, it may have passed to his brother after his death. Regardless, at A. Perceval's meeting with D. Steinhauer and Darlington, the volume passed to her or perhaps even directly to C. Perceval, then a young woman of 14 , who inscribed her name.

In $1828 \mathrm{~A}$. Perceval and the children left Québec to spend a year in Florence. In the autumn of 1829, M. Perceval died at sea en route to join them and the family never returned to Spencer Wood (Pringle 1985). That same year, the Ramsays left Canada, though Christian Ramsay's botanical work continued elsewhere. After this separation from her colleagues in botany, Harriet Sheppard's attentions in natural history turned to ornithology and conchology (Creese 2010).

Catherine Perceval married George William Denys (1811-1881) in 1835 and settled with him at Draycott Hall, Richmond, in Yorkshire having five children (Morgan 1903). George Denys's business was in the local lead mining industry, but by the $1880 \mathrm{~s}$ the mines were worked-out and in debt, and a long series of poor business choices strained the family's fortune (Flynn 1999).
Bookseller's plate: W. Webster. Bookseller \& Stationer, late G. Fell. 60, Piccadilly.

(Fig. 1C).

This bookseller's label would have been in use during the 11 years $1846-57$ by the firm of William Webster, Bookseller \& Stationer, successor to George Fell, in Piccadilly,

London (Brown 1982). It remains uncertain as to what type of service the plates were subjected to. Although we do not know if the plates were previously unbound or not, we assume that the edges were trimmed during binding or re-binding at this time. This resulted in some of the page numbers and last few letters of some of the specific names of illustrated fungi being cut off in some of the plates.

Book plate: Arms, Latin motto: "ORA $E$ SEMPRE”, Sir Francis Denys Bart. (Fig. 1D).

The Denys arms are described in concise heraldic terminology by Debrett (1902). The Latin motto, Ora e sempre ("Now and ever"). Sir Francis Denys Bart. was the second surving son of Catherine and George Denys, Sir Francis Charles Edward Denys (1849-1922), $3^{\text {rd }}$ Baronet (Bart. = Baronet $)$ (Debrett 1902, Lundy 2013). The arms, as well as Francis Denys's name and title, are clues that indicate when the volume came into his possession.

This book plate was printed within the 25 year period 1881-1906: after he inherited the baronetcy from his father, but before he assumed the additional surname of his wife Grace Ellen Burton (18661935 ) and became known as Denys-Burton (Walford 1919, Flynn 1999). The book plate may have been affixed to the volume when he inherited it after his mother's death in 1884. He was the sole executor of her estate (England \& Wales 1858-1966).

We found no records to indicate that Francis Denys had botanical interests. He worked for the British Diplomatic Service and for most of his career he was stationed abroad. Upon inheriting the baronetcy from his father in 1881, he also inherited a "shambolic" state of affairs regarding his father's lead mining interests, from which he continued to try to profit from through 1914 (Flynn 1999). Walford (1919) lists his son Charles Peter (1899-1960) as sole heir. Searches of publicly accessible marriage and death records indicate that Charles married in England (England \& Wales 1916-2005) and died in San Mateo, CA (California
1940-1997). He was the last Denys Baronet (Rayment 2017).

Inscription: "original water-colours of American fungi”. (Fig. 1B).

The next account of the Harvard Icones location and ownership occurs in the late $20^{\text {th }}$ century. It was listed among 114 volumes in a "A collection of works on fungi" for auction in a Christie's catalogue Printed Books: The Salloch Collection: the property of the late William and Marianne Salloch. The auction was held at South Kensington (south-west London) on 31 October 1991. The volume was lot 100: "Original American Watercolours," attributed to Catherine Eliza Perceval (Christie's 1991). The Sallochs, who died within two months of one other in 1991, were partners in life and in the antiquarian book trade for over 50 years. They met at the University of Berlin as history students in the 1930s. Since M. Salloch was of Jewish heritage, they were later expelled from positions in academia and publishing by the Nazi regime. They emigrated to the US, settled in New York, and began prestigious careers as antiquarian book dealers and scholars. They returned yearly to Europe and their native Germany as buyers (Chernofsky 1986, 1990a, b). It is possible that the Harvard Icones was purchased during one of these trips, but we were unable to find substantiating documentation. The Christie's auction catalogue notes that many of the books in the collection of works on fungi bear the personal bookplate of William and Marianne Salloch, however, the Harvard Icones does not. Elsewhere in the catalogue, this collection is specifically attributed to M. Salloch. Most of the books were published volumes containing handcoloured figures. A few notable titles listed with higher starting prices included Batsch's three volume Elenchus Fungorum (178389), the first two volumes of Schäffer's Fungorum qui in Bavaria et Palatinatu circa Ratisbonam (1762-[74]), and Sowerby's three volume Coloured Figures of English Funghi or Mushrooms ([1795-]97-1803[15]). Furthermore, there were three other volumes of original water colours of fungi up for auction along with the Harvard Icones: one from England and two from Japan. The starting price for the Harvard Icones was given at $£ 2-300$ (Christie's $1991)$, and Christie's archives confirms that the hammer price was $£ 260$ ( $£ 286$ inclusive of the Buyer's Premium of $10 \%$ ) 
and the buyer was a book dealer in Bungay (Christie's, pers. comm. 9 Feb. 2015) a small town on the Suffolk coast and about three hour's drive from London.

\section{Book plate: Rare Book S4134. Farlow Reference Library of Cryptogamic Botany. Harvard University. Gift of} Elio Schaechter. Friends of the Farlow. (Fig.1B).

Not long after the auction, shortly prior to 1993, the volume was purchased by Elio Schaechter, a microbiologist, mycophile and first president of the Friends of the Farlow from 1982-85. Schaechter was visting his godson in Norwich, and the pair went to local antiquarian bookstores in search of books on mushrooms. Ultimately they found their way to the nearby Bungay where they found a bookseller with many mycological volumes, but nearly all were out of Schaechter's price range. He settled on the Icones, however, noting the inscription of Philadelphia and realizing that a work relating to fungi of America bearing the early date of 1826 was remarkable. Upon returning to America, he showed the volume to D.H.P., who began to investigate. Cued by the term Niskiensium printed in the title on the volume's cover and recalling the title of the Conspectus, he compared the script of the page numbers and fungal names with handwriting samples by Schweinitz in the collections of the Farlow Reference Library (Schweinitz n.d.) and noted the similarity. Furthermore, the few species marked Nobis were published under the authorship of Albertini and Schweinitz (Schaechter 1997, DH Pfister, pers. comm. 2017). D.H.P. attributed the plates to Schweinitz and generated a manuscript index to the illustrations dated August 1993 and now kept with the volume.

If this narrative of the provenance of the Harvard Icones is accurate, it crossed the Atlantic five times. Presuming that this and the five-volume set were part of Schweinitz's baggage, we may turn to Schweinitz's harrowing account of his return voyage to America in 1812 (Schweinitz 1947, Rogers 1977) for a sense of how fortunate it was that he and his volumes survived. The fate of Michael Perceval at sea (see above), is poignant reminder of the risk taken by those who traveled by ship. On 16 June 1812 , a newly married man, Schweinitz and his bride boarded the Minerva Smyth at Kiel for America. Setting sail from continental European harbours was then stymied by British blockades of Napolean's forces. The British were stopping and searching American ships - often impressing American sailors into their navy, actions that in part drove America to declare war against Britain on 18 June. In an initial attempt to sail, the ship encountered French privateers and was nearly boarded by the British, who were driven off by cannon fire from a nearby Danish fort; the Minerva Smyth had been frightfully postitioned between the guns and their intended targets, the passengers listening to the whistling of cannon balls passing between the masts. After a second, successful attempt, on 8 August the ship was captured in the open sea by the British, but escaped in the night. Having endured several squalls, on 26 August a hurricane nearly capsized the ship. Water poured into the cabin. The passengers had resigned themselves to the fate that they felt must surely come, but the ship was righted and all survived. The next day calm weather allowed for repairs to the severely damaged vessel. While off the coast of Rhode Island, having missed a flotilla of British warships by three days, the Minerva Smyth safely archored at New York on 8 September, and the Schweinitz's arrived in Bethlehem (PA). Their journey to America had taken three months and twelve days.

\section{On the Harvard Icones as a sketchbook, created early in the development of the Conspectus}

While conducting research for his 2002 paper, Hewitt observed a number of distinguishing characteristics of the Harvard Icones in relation to the four volumes of the five-volume set held at ANS. The ANS volumes differed in the larger sized format and having title pages. The plates of the Harvard Icones are numbered 1-50 and these plate numbers are also in volume 1 at ANS. Finally, at least one illustration in the Harvard Icones is duplicated in one of the four ANS volumes. These observations led Hewitt to conclude that the Harvard Icones was not part of the five-volume set. Furthermore, when he assessed the quality of the figures in the Harvard Icones as biological illustrations, he found that many of them were rudimentary or incomplete. He hypothesized that the Harvard Icones served Schweinitz as a sketchbook during the early years of the development of the Conspectus (D. Hewitt, pers. comm. Aug. 2000). Our observations support this hypothesis and the remainder of this section expands on this view. We begin with an assessment of the figures in the Harvard Icones as biological illustrations followed by a discussion on the various notations that Schweinitz affixed to some of these. We then describe how two new species of fungi illustrated in this volume began as provisionally named, preliminary sketches and ended as published names with finished illustrations in the five-volume set and Conspectus. Finally, we conclude with a brief discussion of the seasonality of the fungi depicted in the Harvard Icones.

A representative plate from the Harvard Icones is adapted in Fig. 2. Here, the elements of each figure are composed in a stereotypical way. This allows the viewer to quickly grasp distinguishing characteristics of each species. For example, the caps of one mushroom in each figure are oriented toward the viewer in order to show details of colouration and surface texture. In the other mushrooms, the cap is tilted away from the viewer so that these details can be observed in the gills and stipe. The insets each show a mushroom in longitudinal section through the centre, so that the viewer may observe the way that the gills attach to the stipe and whether the tissue of the stipe is hollow. Although this is helpful information, the figures are incomplete as the bases of the mushrooms are not shown. As a result, important information regarding their morphology, as well as from what type of substratum the mushroom is emerging, is not provided. Finally, in a finished plate, it is advantageous for a biological illustrator to group several figures of closely related or morphologically similar species together in order to facilitate comparison. This was not done in the Harvard Icones plates. In the example presented in Fig. 2, the plate comprises figures of morphologically distinctive Agaricus species from sections Lepiota, Lactifluus, and Gymnopus. These paintings are quick sketches, and appear to have been executed on each page roughly in the order that the specimens were collected, as often done when making field-notes. No attempt was made to group closely related species on a plate or over a number of consecutive plates. In contrast, Schweinitz systematically arranged the completed illustrations in the five-volume set into plates following Persoon's (1801) classification.

Although many of the figures in the Harvard Icones are rudimentary, a small 

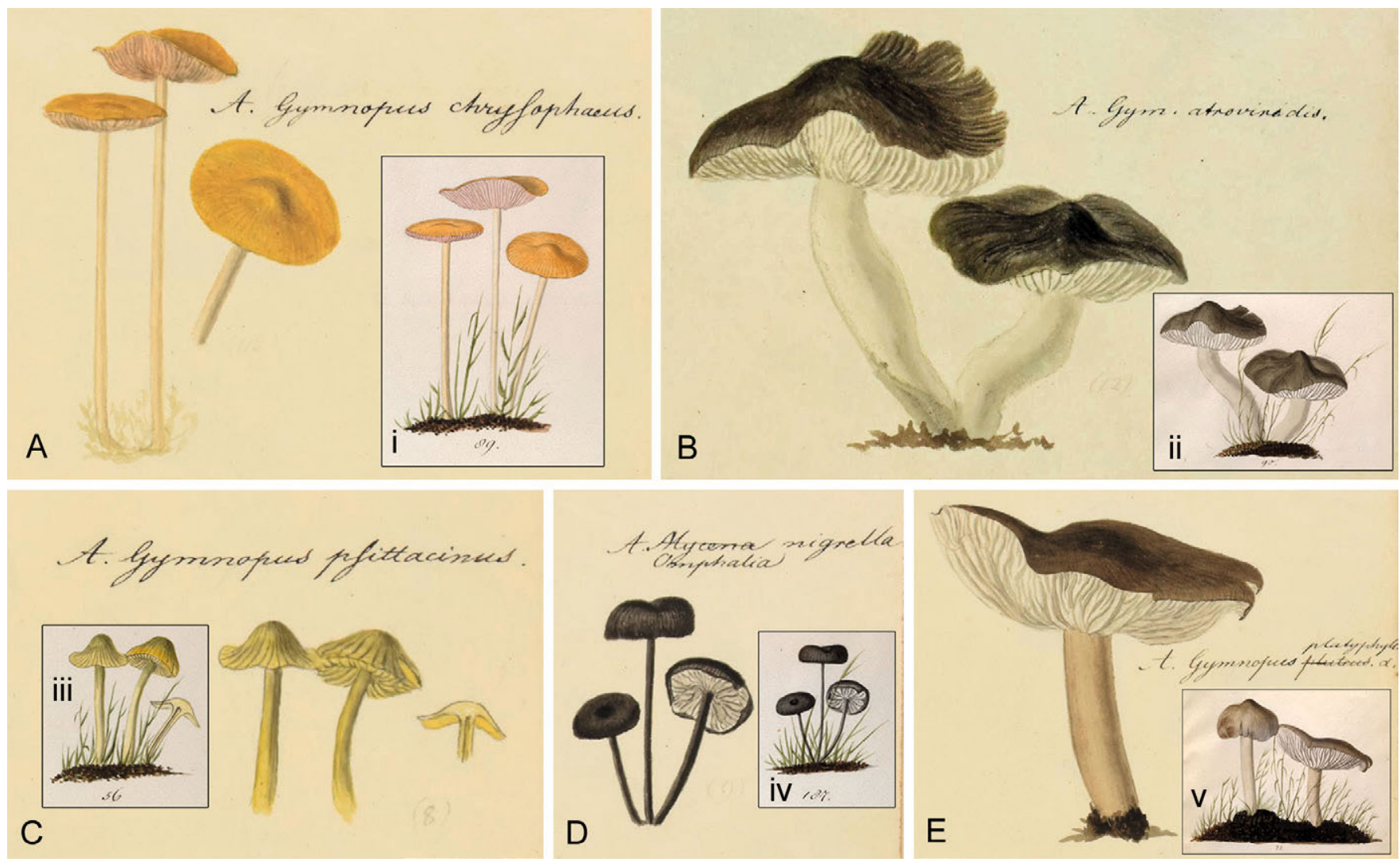

Fig. 3. Examples of figures from the Harvard Icones that were copied by Schweinitz into finished illustrations in the five-volume set (shown in the inserts i-v). All are in water colour. A. Agaricus (Gymnopus) chrysophaeus (Harvard Icones plate 7; insert i: plate 116, figure 89 [probably = A. G. leoninus var. $\beta$, chrysophaeus in the Conspectus]). B. Agaricus (Gymnopus) atrovirens (Harvard Icones plate 46; insert ii: plate 117, figure 90 as A. G. luridus). C. Agaricus (Gymnopus) psittacinus (Harvard Icones plate 47 (see Fig. 2 above); insert iii: plate 104, figure 56). D. Agaricus (Omphalia) nigrella (Harvard Icones plate 50; insert iv: plate 153, figure 187). E. Agaricus (Gymnopus) platyphylla $\alpha$ (Harvard Icones, plate 23; insert v: plate 113, figure 77). It is currently unknown what the numbers, “(8)” and “(9)” refer to in C and D, respectively. Images from the Harvard Icones courtesy of the Farlow Reference Library of Cryptogamic Botany, Harvard University. Inserts i-v adapted from Fungorum Nieskiensium Icones, volume 2, courtesy of the library and archives of The Academy of Natural Sciences of Drexel University, Philadelphia, Pennsylvania.

number of them were perhaps Schweinitz's only record of certain collections of fleshy fungi in the living state, and these were directly copied into finished illustrations in the five-volume set. As noted above, Hewitt (pers. comm. Aug. 2000) discovered one case of this, and we detected a few more; these examples are presented in Fig. 3.

That the Harvard Icones was a work-inprogress is also reflected in the various edits and annotations to many of the scientific names that Schweinitz appended to the figures. As seen in Fig. 2, some illustrations are of undetermined taxa with no names. In others, a name is written faintly in pencil, indicating that the determination was tentative. This is in contrast to a name written in ink, which is an indication of a more confident determination. In a number of cases, names written in either pencil or ink are struck through and another name is inserted. Some illustrations are of subjects that were morphologically anomalous and labeled "monstrosa". Examples include Clavaria monstrosa (Pl. 20 and 30), and
Naemaspora crocea monstrosa (Pl. 27). Other notations are applied to particular illustrations to indicate uncertainty as to whether the specimen is even a fungus. A taxon depicted on Plate 28 is labeled "Inexplicatus Fungus" and Merulius crispus on Plate 49 is labeled "inexplicat [us]". An illustration of an unnamed dark green gelatinous substance, labeled "Substantia aquatica" [watery substance] (possibly Nostoc?), is also depicted on Plate 49. Question marks are affixed to many determinations throughout the volume. In contrast, there are no such curiosities or ambiguities in the five-volume set. Other notations in the Harvard Icones indicate the state or quality of the specimen from which the illustration was made: Boletus citrinus on Plate 13 is labeled "obsolete" [senescent], Trichia rubiformis on Plate 6 is labeled "statu jun." [immature state], and Merisma foetidum on Plate 17 is labeled "spec. parv." [meager specimen]. Varieties or noteworthy colourations are also noted. All of these notations are transcribed verbatim in Table 2. Non-consecutive numbers in parentheses are written in faint pencil on many of the figures; what these refer to is unknown. They do not correspond to numbered taxa in Persoon (1801) or to any of the other volumes that Albertini and Schweinitz referenced in the development of the Constpectus (Hewitt et al. 2016). If they are collection numbers, they do not refer to fungal specimens that belonged to Albertini and that are now in the collections of the Royal Botanic Gardens Victoria in Melbourne, Australia (MEL). These specimens were recently digitized and made available through Global Plants on JSTOR (T. May, pers. comm.); there are no collector numbers associated with these specimens.

The cases of two figures in the Harvard Icones, designated "Nobis", further demonstrate how this volume served as an early sketchbook. This is evidenced by a comparison of the sketch-like qualities of the illustrations and the provisional names assigned to them, with the finished illustrations and formal names given in 

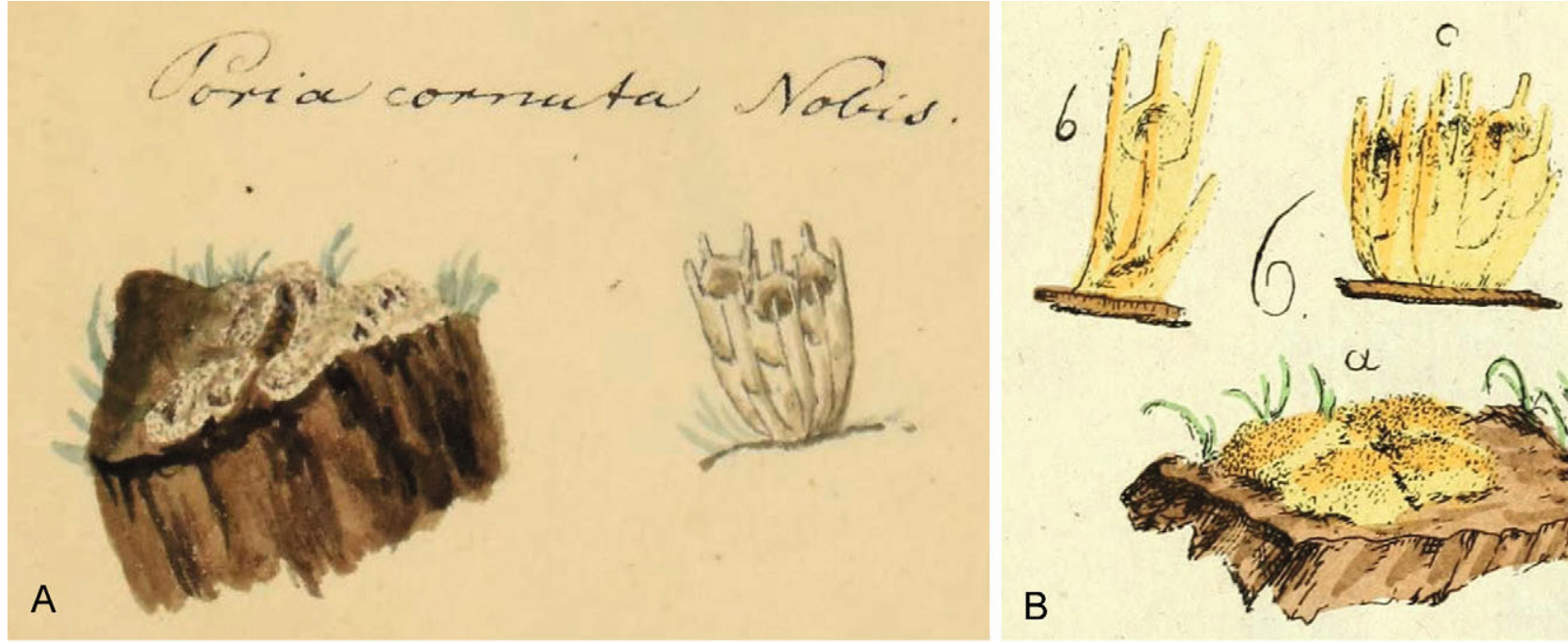

B
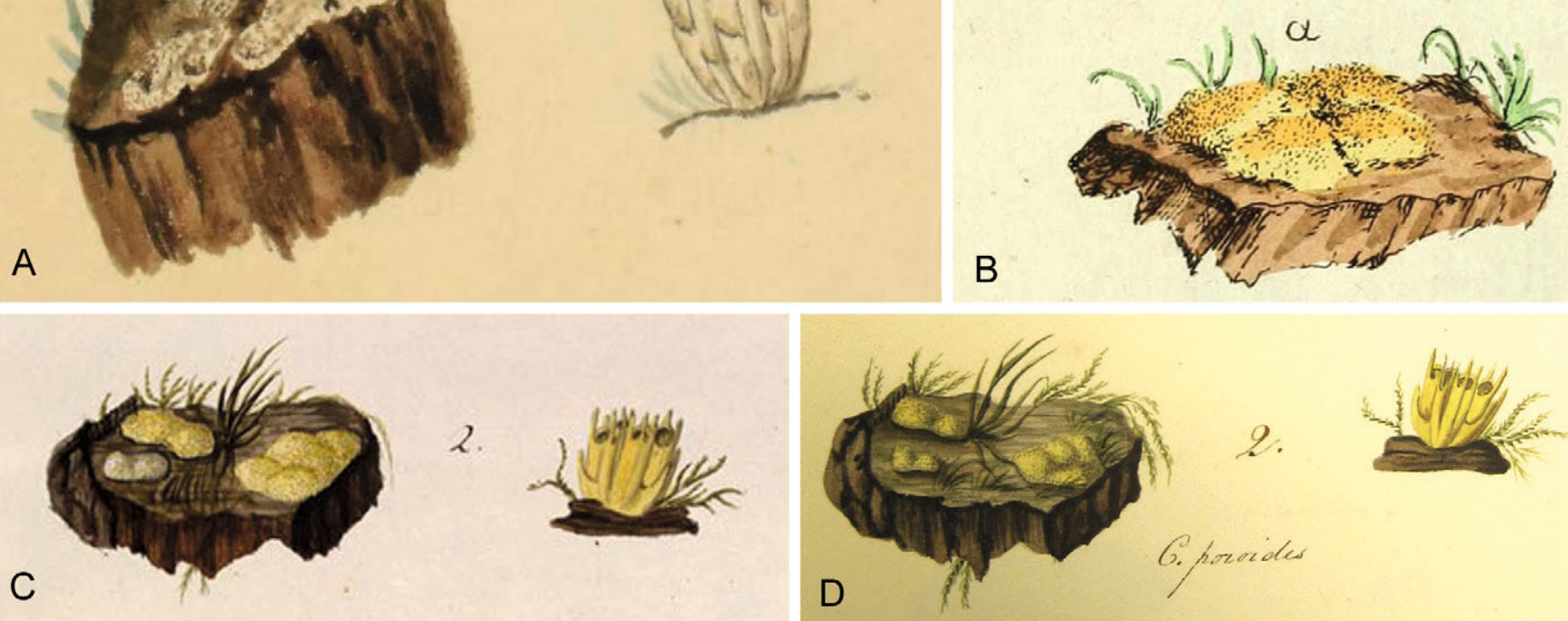

Fig. 4. A comparison of figures depicting Ceratium porioides var. $\beta$, flavum Albertini \& Schweinitz. All are in water colour except B that is a hand-coloured etching. A. Poria cornuta, "Nobis" (Harvard Icones plate 21). Image courtesy of the Farlow Reference Library of Cryptogamic Botany, Harvard University. B. Ceratium porioides var. $\beta$, flavum (Conspectus plate 2, figure 6). In the legend for this figure, given in the Explicatio iconum, Albertini and Schweinitz demur: NB. Icon haec sequensque minus idoneae [Note well. This icon and the following are less than ideal]. Image courtesy of the New York Botanical Garden, LuEsther T. Mertz Library. C. Ceratium poroides (volume 5 of the five-volume set, plate 429, figure 2). Image courtesy of the library and archives of The Academy of Natural Sciences of Drexel University, Philadelphia, Pennsylvania. D. Ceratium poroides (Icones Fungorum plate 286, a copy of C). Image courtesy of the botanical library of the University of Michigan, Ann Arbor.

the five-volume set and published in the Conspectus.

(1) Ceratium porioides var. $\beta$, flavum Alb. \& Schwein. (now Ceratiomyxa fruticulosa f. flava (Alb. \& Schwein.) Y. Yamam.) is a slime mould not a true fungus (Fig. 4). In the Harvard Icones, the illustration depicting this taxon is labeled Poria cornuta "Nobis" (Fig.4A). In this figure, the element that shows the habit of the organism on wood is hastily painted and muddled when compared to the finished illustrations in the Conspectus (Fig. 4B) and in volume 5 of the five-volume set (Fig. 4C). This latter illustration is labeled Ceratium poroides, an orthographic variant of $C$. porioides, and a copy of this figure is similarly labeled in the Michigan Icones (Fig. 4D).

The name change from Poria cornuta, as given in the Harvard Icones, to the published name $C$. porioides, reflects the evolution of Albertini and Schweinitz's concept of the organism over time. In gross morphology, the organism depicted in these figures is somewhat effuse, tightly attached to a piece of wood, and seems to have a poroid surface, like species of Poria. When the specimen was first collected and the figure painted in the Harvard Icones, Albertini and Schweinitz understood it to be a new species in this genus and so named it Poria cornuta. In time, they reconsidered this and published it under their new generic name, Ceratium, as $C$. porioides - the "Poria-like" Ceratium. The genus is classified among the Naematothecii, a heterogeneous assemblage of slime moulds (protists) and true fungi with web-like, mould-like or otherwise reduced gross morphology, rather than among Poria, then considered a group in Boletus, that produce conspicuous mushrooms with the spores generated within pores.

(2) Peziza rhizopus Alb. \& Schwein. (now Plectania melastoma (Sowerby) Fuckel). This is the only taxon that is illustrated in the Harvard Icones, the Conspectus, the five-volume set, and the Michigan Icones (Fig. 5). It is listed in [Schweinitz] (n.d.) as having been collected in autumn 1801. The rudimentary depiction of this taxon in the Harvard Icones bears few characters in common with the more detailed representations, other than a cupulate habit and a dentate margin. The evolution of its name also serves to date the Harvard Icones as originating early in the development of the Conspectus. When the specimen was first illustrated in the Harvard Icones, it was labeled with the provisional name $P$. rhizophora (Fig. 5A). It is similarly named in volume 3 of the fivevolume set (Fig. 5B) and in [Schweinitz] (n.d.). According to a translation of parts of Schweinitz's diary that relate to the Conspectus, volume 3 was completed on 14 May 1803 (Schweinitz 1946). The taxon was then published as P. rhizopus in the Conspectus in 1805 (Fig. 5C). Finally, when the figure was copied from volume 3 of the 5-volume set to make up part of Pl. 255 in the Michigan Icones, it was labeled $P$. rhizopus (Fig. 5D).

A consideration of the seasonality of the fungi illustrated in the Harvard Icones indicates that the volume treats those species that occur in summer and fall. For example, on plate 1 an Amanita species is depicted. On plate 3 an Otidea species and a clearly mature specimen of Sphaeria 

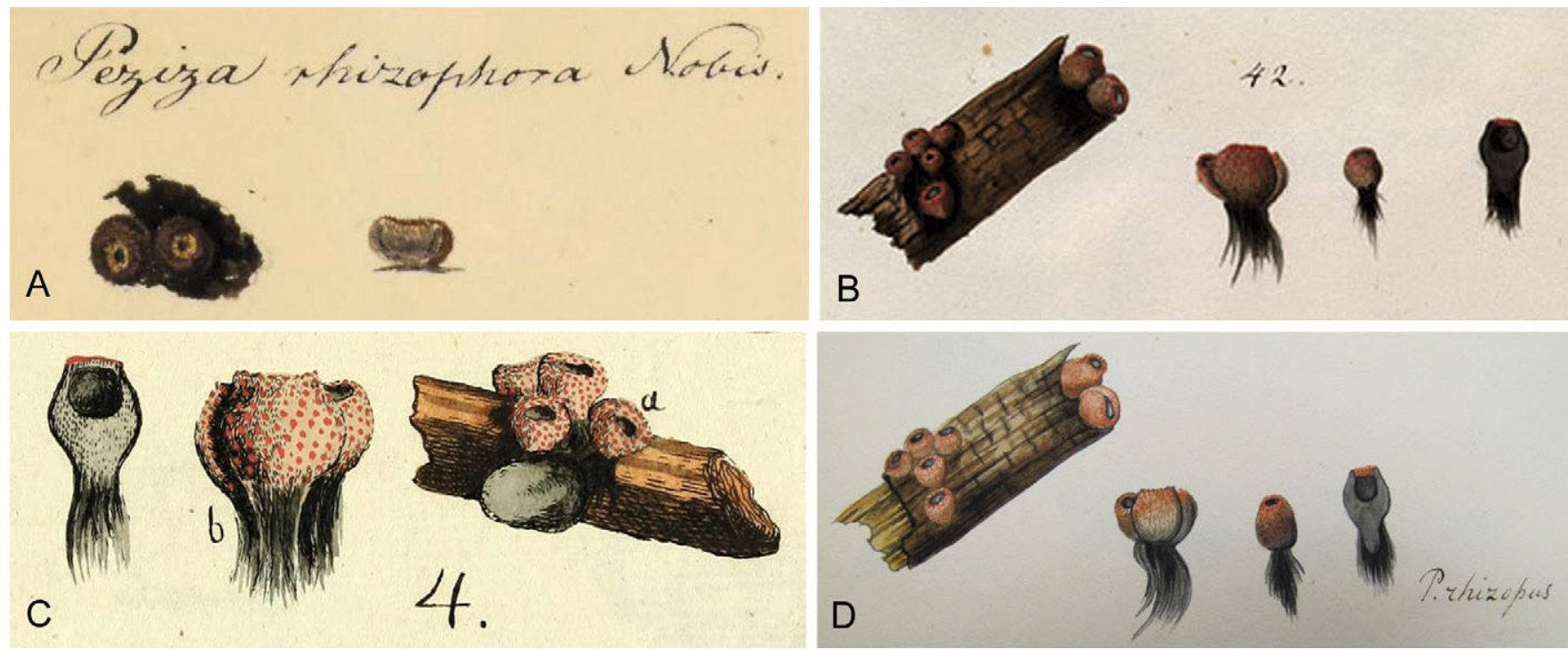

Fig. 5. A comparison of figures depicting Peziza rhizopus Albertini \& Schweinitz. All are in water colour except C that is a hand-coloured etching. A. Peziza rhizophora, "Nobis" (Harvard Icones, plate 28). Image courtesy of the Farlow Reference Library of Cryptogamic Botany, Harvard University. B. Peziza rhizophora (volume 3 of the 5-volume set, plate 250, figure 42). Image courtesy of the library and archives of the Academy of Natural Sciences of Drexel University. C. Peziza rhizopus (Conspectus plate 1, fig. 4). Image courtesy of the New York Botanical Garden, LuEsther T. Mertz Library. D. Peziza rhizopus (Icones Fungorum, plate 255, a copy of B). Image courtesy of the botanical library of the University of Michigan, Ann Arbor.

bypoxylon (now Xylaria bypoxylon) are illustrated. Mature specimens of the latter are commonly found in late summer into the fall. As far as we are able to determine, there are no springtime fungi such as Morchella, Gyromitra, or Mitrula species depicted on any of the plates, with the possible exception of an unnamed, stipitate, ascomycete-like fungus on Pl. 24 that could be Vibrissea truncorum (as Leotia truncorum in Albertini \& Schweinitz 1805: 297, Pl. 3 fig. 2) that is listed in the Conspectus as occurring in May and June. It is not immediately apparent that the plates are in a chronological order, with figures on earlier plates depicting collections found in mid-summer, and later plates depicting those found in late-summer and into fall. It is also unclear as to whether the plates were all painted within the same year.

\section{SUMMARY}

The Conspectus has been an important mycological work, recently recognized not only for its nomenclatural importance as a naming volume but also as a window into the ecology of the region around Niesky. Nonetheless, only now has the background of the production of its illustrations been addressed. That various volumes of illustrations have been dispersed has made the task more difficult. Notable among our findings has been the identification of the Harvard Icones as a working record of Albertini and Schweinitz's research. That it survived in private hands for more than 170 years and found its way to a permanent home is remarkable, and no less so is the fact that, largely in an era of ships' crossings, the volume made at least five trans-Atlantic crossings. This along with the recent discovery of extant Albertini and Schweinitz specimens in Melbourne (MEL) opens further research possibilities on Schweinitz. Finally, it is important to note that much of this work that resulted in this paper was made possible because archives, manuscripts and books are becoming increasingly available through digital efforts being made around the world.

\section{ACKNOWLEDGEMENTS}

We first thank anonymous reviewers. Also David Hewitt for sharing with us his research notes and observations stemming from his 2002 publication; Michaela Schmull for translation work from German to English of the Conspectus review (Anon. 1806); Judith Warnement, Diane Rielinger and staff at Harvard University Botany Libraries for digitizing the Harvard Icones; Jennifer Vess and staff at the archives of the Academy of Natural Sciences of Drexel University for their work in digitizing volumes $1-3$, and 5 of the five-volume set and for their assistance with the Schwienitz papers; Tammy Kiter and staff at the New York Historical Society, Manuscripts Department; Michelle Fitch at West Chester University, Special Collections and Archives; Christiane Anderson, Patricia Rogers and staff at the University of Michigan, Ann Arbor, Herbarium Library; Meghan Constantinou and staff at The Grolier Club; Andrew Hart, Jason Tomberlin, Frederick Stipe and staff at the University of North Carolina, Chapel Hill, Wilson Library for digitizing volume 4 of the five-volume set; and Tom May, Senior Mycologist at the Royal Botanic Gardens Victoria, Melbourne, Australia, for bringing to our attention the existence of Albertini and Schweinitz's Niesky fungi in their collections, and for his efforts in making them available in digitized form through JSTOR Global Plants.

\section{REFERENCES}

Albertini JB de, Schweinitz LD de (1805) Conspectus fungorum in Lusatiae superioris agro Nieskiensi crescentium. E methodo Persooniana. Leipzig: Kummerlan. [New York Botanical Garden, LuEsther T. Mertz Library. Retrieved from https://doi.org/10.5962/bhl.title.3601.]

Anon. (1806) Jenaische Allgemeine Literatur-Zeitung 62: 492-496.

Batsch AJGC (1783-89) Elenchus fungorum. Halle: Joannem J. Gebaver.

Brown PA (1982) London Publishers and Printers, $c$. 1800-1870. London: The British Library.

California, Death Index, 1940-1997. [Database on-line. Original data: State of California. California Death Index, 1940-1997.

Sacramento, CA: Department of Health Services, Center for Health Statistics.] 
Chernofsky JL (1986) Sharing in the pursuit of books and scholarship. Antiquarian Booksellers Weekly (8 Dec.): 2333-2335.

Chernofsky JL (1990a) AB obituary notes: Marianne [Blum] Salloch. Antiquarian Booksellers Weekly (1 Jan.): 12.

Chernofsky JL (1990b) AB Obituary Notes: William [Siegfried] Salloch. Antiquarian Booksellers Weekly (12 March): 1121.

Christie's (1991) Printed Books: the Salloch Collection. [Auction no. 4458.] London: Christie's.

Creese M (2010) Ladies in the Laboratory III. Lanham, MD: Scarecrow Press.

Darlington, W. (1826) Letter to Anne Mary Perceval. 10 June 1826. [Dr. William Darlington Papers, Letterbook: Correspondence with Various Persons, Vol. 1 (1805-1837), West Chester University, Special Collections, Francis Harvey Green Library, PA.]

Debrett (1902) Debrett's Peerage, Baronetage, Knightage, and Companionage. Royal edn. London: Dean \& Son.

England \& Wales, National Probate Calendar (Index of Wills and Administrations), 1858-1966. [Database on-line. Original data: Principle Probate Registry. Calendar of the Grants of Probate and Letters of Administration made in the Probate Registries of the High Court of Justice in England.]

England \& Wales, Civil Registration Marriage Index, 1916-2005. [Database on-line. Original data: General Register Office. England and Wales Civil Registration Indexes

Flynn CG (1999) The decline and end of the lead mining industry in the northern Pennines 18651914: a socio-economic comparison between Wensleydale, Swaledale and Teesdale. MA thesis, Durham University. [http://etheses.dur. ac.uk/4569/.]

Hamilton JT (1900) A history of the church known as the Moravian Church, or The Unitas Fratrum, or The Unity of the Brethren, during the eighteenth and nineteenth Centuries. Transactions of the Moravian Historical Society 6.

Hewitt D (2002) Lewis David von Schweinitz's mycological illustrations. Bartonia 61: 48-53.

Hewitt D, Amram P, Schmull M, Karakehian JM (2016) An early mycota: Johannes Baptista von Albertini and Lewis David von Schweinitz's Conspectus fungorum in Lusatiae superioris agro Niskiensi crescentium, with a translation of the Latin introduction into English. Bartonia 69: 47-61.

Johnson WR (1835) A memoir of the late Lewis David von Schweinitz, P.D. with a sketch of his scientific labors. [Read before the Academy of Natural Sciences of Philadelphia, 12 May 1835.]
Keeney EB (1992) The Botanizers: amateur scientists in nineteenth-century America. Chapel Hilll, NC: University of North Carolina Press.

Krieger LCC (1938-1939) Unpublished plates of fungi by Lewis David von Schweinitz. Bartonia 20: $28-29$.

LeMoine JM (1882) Picturesque Quebec: a sequel to Quebec Past and Present. Dawson Bros.

Lundy D (2013) Sir Francis Charles Edward Denys-Burton, $3^{\text {rd }}$ Bt. Person page no 19441 [Retrieved from The Peerage: a genealogical survey of the peerage of Britain as well as the royal families of Europe. http://www.thepeerage.com/ p19441.htm.]

Lynch DM (1996) Paintings of fungi by Lewis David von Schweinitz in the archives of the Academy of Natural Sciences of Philadelphia. Bartonia 59: 125-128.

Morgan H (ed.) (1903) Types of Canadian Women. Vol. 1. W. Briggs.

Perceval AM (n.d.) Letter to John Torrey. [John Torrey Papers, Series 1 (correspondence), Box 6, Folder 6.64. Archives of the New York Botanical Garden.]

Perceval AM (1826) Letter to William Darlington. 6 Oct. 1826. [William Darlington Papers, Correspondence 1800-1863, Vol. 4. Patricia D. Klingenstein Library, New York Historical Society.]

Persoon CH (1801) Synopsis Methodica Fungorum. Göttingen: Henricus Dieterich.

Pringle J (1985) Anne Mary Perceval (1790-1876), an early botanical collector in lower Canada. Canadian Horticultural History 1: 7-13.

Rayment L (2017) Baronatage: DENYS of Stratford Place, London. 18 Aug. [Retrieved from Leigh Rayment's Peerage Page. http://www. leighrayment.com/baronetage/baronetsD2. $\mathrm{htm} /]$

Rogers DP (1977) L. D. de Schweinitz and early American mycology. Mycologia 69: 223-245.

Schaechter E (1997) In the Company of Mushrooms: a biologist's tale. Cambridge, MA: Harvard University Press.

Schäffer JC (1762-[74]) Fungorum qui in Bavaria et Palatinatu circa Ratisbonam nascuntur icones nativis coloribus expressae. Regensburg.

Schweinitz LD von (n.d.) Conspectus muscorum frondosorum americanorum in herbario adjectis ceteris ab aliis in America observatis:Musci hepaticae americani herbarii. Diarium cryptogamiae, Jan.-Mar. 1821. [Manuscripts, Farlow Reference Library of Cryptogamic Botany, Harvard University.]

[Schweinitz LD von] (n.d.) Fungi nostrates novi: aut species, aut varietates tantum aut denique characteribus novis ditatas Persoonii fungos complectens. Clariss. v. Albertini recensuit. [Our new fungi: or species, or only varieties or finally new characters to enrich the fungi included in Persoon. Enumerated by the renowned v. Albertini. Includes collection dates.] [Academy of Natural Sciences of Drexel University, Philadelphia, Pennsylvania, Academy Library and Archives. L. D. von Schweinitz papers, Collection no. 4 "miscellanea," Folder 1.]

Schweinitz LD von (ca. 1802-03) Fungorum Nieskiensium Icones. [Volume 1.] [Academy of Natural Sciences of Drexel University, Philadelphia, Pennsylvania, Academy Library and Archives. L. D. von Schweinitz papers, Collection no. 437. https://archive.org/ details/fungorumnieskie1schw:] https://www. biodiversitylibrary.org/item/247183\#page/1/ mode/lup.

Schweinitz LD von (ca. 1802-03) Fungorum Nieskiensium Icones. [Volume 2.] [Academy of Natural Sciences of Drexel University, Philadelphia, Pennsylvania, Academy Library and Archives. L. D. von Schweinitz papers, Collection 437. https://archive.org/details/ fungorumnieskie2schw.] https://www. biodiversitylibrary.org/item/247134\#page/1/ mode/lup.

Schweinitz LD von (1803) Fungorum Nieskiensium Icones. [Volume 3.] [Academy of Natural Sciences of Drexel University, Philadelphia, Pennsylvania, Academy Library and Archives. L. D. von Schweinitz papers, Collection no. 437. https:// archive.org/details/fungorumnieskie3schw.] https://www.biodiversitylibrary.org/ item/247253\#page/1/mode/lup.

Schweinitz LD von (ca. 1803-05) Fungorum Niskiensium Icones. [Volume 4.] University of North Carolina, Chapel Hill, Rare Book Collection, Wilson Library. http://www. biodiversitylibrary.org/bibliography/122159\#/ details. https://archive.org/details/ Fungorum 1805Schweinitz.].

Schweinitz LD von (ca. 1803-05) Fungorum Niskiensium Icones. [Volume 5.] [Academy of Natural Sciences of Drexel University, Philadelphia, Pennsylvania, Academy Library and Archives. L. D. von Schweinitz papers, Collection no. 437. https://archive.org/ details/fungorumnieskie5schw.] https://www. biodiversitylibrary.org/item/247262\#page/1/ mode/lup.

Schweinitz LD von (ca. 1798-1802) Icones Fungorum Niskiensium. [Farlow Reference Library of Cryptogamic Botany, Harvard University. https://doi.org/10.5962/bhl. title.137760.]

Schweinitz LD von (ca. 1818-26) Icones Fungorum.

[University of Michigan, Ann Arbor,

Herbarium Library. A manuscript index prepared by L. C. C. Krieger is kept with this volume.]

Schweinitz LD von (1825) Letter to William Darlington. 6 March. [William Darlington 
Papers, 1788, [ca. 1800-63, Correspondence 1800-1863, Volume 8. Patricia D. Klingenstein Library, New York Historical Society.]

Schweinitz LD von (1946) Preparation of the "Conspectus Fungorum Lusatiae" by Lewis David de Schweinitz and John Baptist von Albertini. Taken from the diaries of L. D. von Schweinitz, on file in the Salem Moravian Archives. [Years 1801 and 1803]. Translated by Adelaide L. Fries, Archivist for the Moravian Church in America, Southern Province. [Copy on file at University of North Carolina, Chapel Hill, Botany Department Papers, Southern Historical Collection, No. 2053, Folder 143. With cover letter from A. L. Fries to W. C. Coker dated 7 Nov. 1946.]

Schweinitz LD von (1947) Account of the journey of Br. Ludwig von Schweinitz and his wife from Herrnhut to Bethlehem in Pennsylvania, from June 4 to September 16, 1812. Translated by A. L. Fries [Diary in the Salem Moravian Archives]. In Records of the Moravians in North Carolina (Fries AL ed.) 7: 3021-3043.

Shear CL, Stevens NE (1917) Studies of the Schweinitz collections of fungi - I. Sketch of his mycological work. Mycologia 9: 191-204.

Sowerby J ([1795-]97-1803[-15]) Coloured Figures of English Funghi or Mushrooms. London: Sowerby.

Steinhauer H (1810) Letter to James Edward Smith of Norwich, [Norfolk]. 5 Sept. [The Linnean Society of London. Ref No GB-110/JES/ ADD/99. http://linnean-online.org/64486/.] Steinhauer H (1818) Letter to John Eatton Leconte. 21 Jan. [Herbarium Library, University of Michigan, Ann Arbor.]
Stuckey RL (1966) Daniel Steinhauer, early Ohio plant collector, and his correspondence with the botanist Schweinitz. Bartonia 36: 1-24.

Torrens HS (2005) The Moravian Minister Rev. Henry Steinhauer (1782-1818): his work on fossil plants, their first 'scientific' description and the planned Mineral Botany. In History of Paleobotany: selected essays (A Bowden, C Burek, R Wilding, eds) 241: 13-28. London: Geological Society of London.

Walford E (1919) Walford's County Families of the United Kingdom. London: Ballantyne.

Wilbert MI (1908) Some early botanical and herb gardens. American Journal of Pharmacy 80 : 412-427. 